\title{
A unified Lagrangian formulation for solid and fluid dynamics and its possibility for modelling submarine landslides and their consequences
}

\author{
Xue Zhang ${ }^{\mathrm{a}, \mathrm{b}, *}$, Eugenio Oñate ${ }^{\mathrm{b}}$, Sergio Andres Galindo Torres ${ }^{\mathrm{a}}$, Jeremy Bleyer ${ }^{\mathrm{c}}$, \\ Jeremy Bleyer ${ }^{\mathrm{a}}$ \\ ${ }^{a}$ Department of Civil Engineering and Industrial Design, University of Liverpool, Liverpool, United Kingdom \\ ${ }^{\mathrm{b}}$ International Centre for Numerical Methods in Engineering (CIMNE), Barcelona, Spain \\ ${ }^{\mathrm{c}}$ Université Paris-Est, Laboratoire Navier (ENPC, IFSTTAR, CNRS UMR 8205), Champs-sur-Marne, France
}

\begin{abstract}
Consequences of submarine landslides include both their direct impact on offshore infrastructure, such as subsea electric cables and gas/oil pipelines, and their indirect impact via the generated tsunami. The simulation of submarine landslides and their consequences has been a long-standing challenge majorly due to the strong coupling among sliding sediments, seawater and infrastructure as well as the induced extreme material deformation during the complete process. In this paper, we propose a unified finite element formulation for solid and fluid dynamics based on a generalised Hellinger-Reissner variational principle so that the coupling of fluid and solid can be achieved naturally in a monolithic fashion. In order to tackle extreme deformation problems, the resulting formulation is implemented within the framework of the particle finite element method. The correctness and robustness of the proposed unified formulation for single-phase problems (e.g. fluid dynamics problems involving Newtonian/Non-Newtonian flows and solid dynamics problems) as well as for multi-phase problems (e.g. two-phase flows) are verified against benchmarks. Comparisons are carried out against numerical and analytical solutions or experimental data that are available in the literature. Last but not least, the possibility of the proposed approach for modelling submarine landslides and their consequences is demonstrated via a numerical experiment of an underwater slope stability problem. It is shown that the failure and post-failure processes of the underwater slope can be predicted in a single simulation with its direct threat to a nearby pipeline and indirect threat by generating tsunami being estimated as well.
\end{abstract}

Keywords: Submarine landslide; Unified FE formulation; Monolithic coupling; Fluid-solid interaction; Mathematical programming; PFEM

\footnotetext{
* Corresponding author. Department of Civil Engineering and Industrial Design, University of Liverpool, Liverpool, United Kingdom. E-mail address: xue.zhang2@liverpool.ac.uk (X. Zhang).
} 

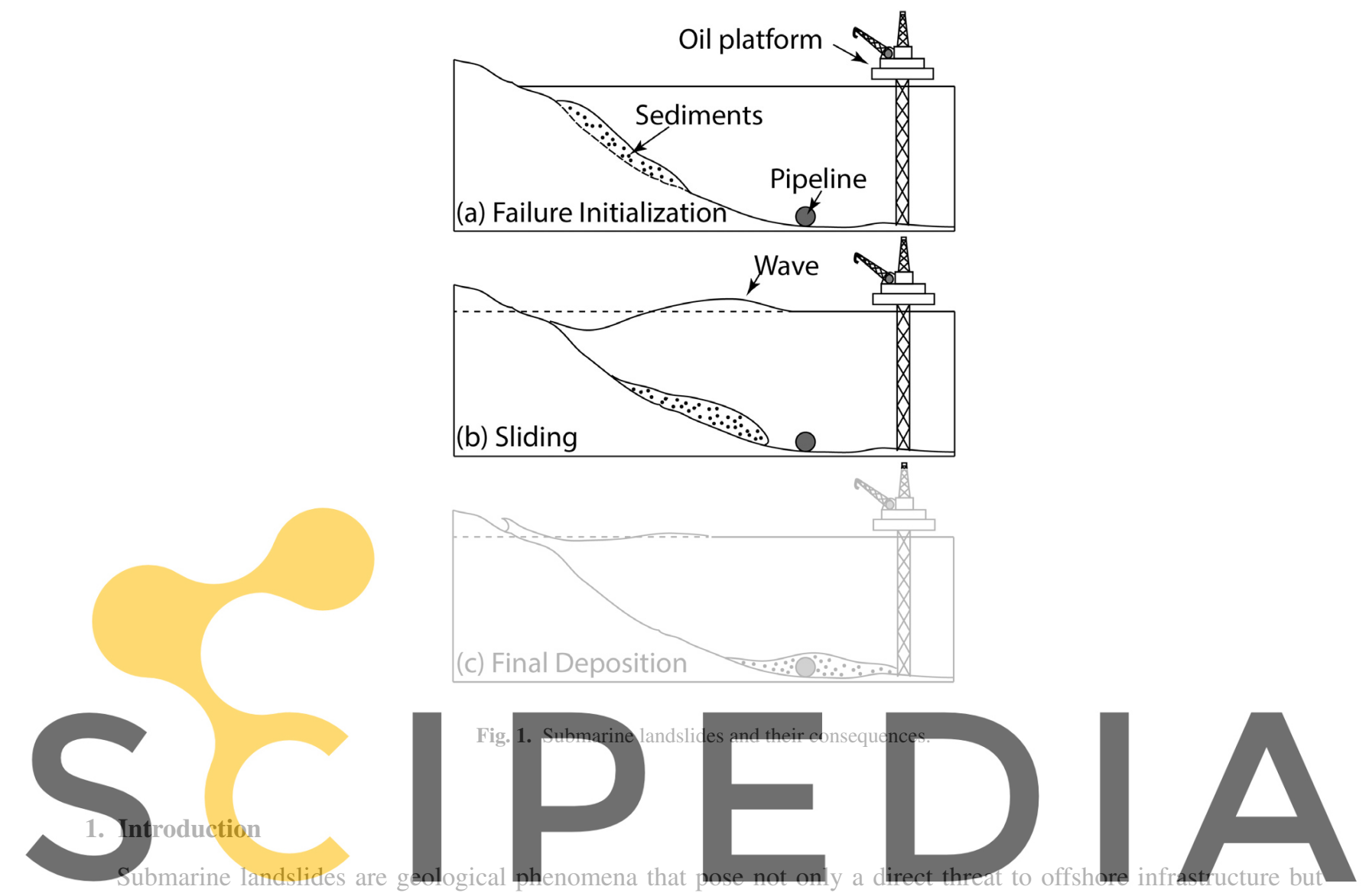

also an indirect threat to coastal communities through the generation of tsunamis. Typical examples are the $1998 \mathrm{New}$

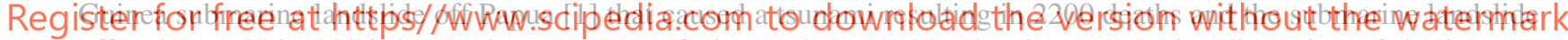
off Taiwan [2] in 2006 that broke seven out of nine undersea cables leading to a major disruption of the internet connection and general commerce between Thailand, Malaysia, Vietnam, South Korea, China and Singapore. In the past decade, submarine landslides have been receiving increasing attention which is, to a large extent, due to a boom in offshore infrastructures such as submarine gas and oil pipelines, offshore wind farm and electricity grid infrastructure, deep-water oil and gas platforms etc.

The timely forecast of a potential submarine landslide, as well as a realistic estimation of its post-failure behaviour and consequences, is undoubtedly of great significance for minimising the degree of destruction. Conventional geotechnical approaches, such as the limit equilibrium method, the limit analysis method and the displacement-based finite element method that are widely used for slope stability analysis normally stop at the point when failure is triggered and do not provide information regarding the post-failure process. To forecast a submarine landslide and estimate its potential impacts, ideally the complete process of the slide ranging from its failure initiation through migration to its final deposition is produced via a single simulation seamlessly. This task however is formidable due to the complex coupling mechanism involved in the process as well as the solid-fluid transitional behaviour of the evoked submarine soil mass.

In a submarine landslide, the sediment behaves like a solid before the slide is initiated (Fig. 1(a)) and after the sliding mass eventually comes to rest at a new location (Fig. 1(c)), but mimics a fluid during the sliding process (Fig. 1(b)). When the post-failure stage is concerned, the sliding sediment is commonly simulated based on the framework of fluid mechanics, due to its fluid-like behaviour. In the simulation, the sediment is treated as a non-Newtonian flow while the seawater as a Newtonian flow, both solved according to either Navier-Stokes equations $[3,4]$ or simplified governing equations such as the shallow water theory [5,6]. Despite the prevalence of this solution strategy (particularly for modelling submarine landslide generated tsunami), it fails to capture the solid-like features of subsea sediments and thus does not perform well for the stability analysis of underwater slopes or for the analysis 
of their progressive failure behaviour. Recent efforts made in this regard include [7-9] in which simulations were carried out in the framework of solid (or soil) mechanics. Owing to the low permeability, marine clays in these works were represented by the Tresca or Von-Mises constitutive model implying an undrained condition. The progressive development of plastic shear deformation in marine clays was reproduced via the reduction of undrained shear strength with accumulated plastic displacement or strain. Influence of seawater on the submarine landslides in [7-9] was considered by using the submerged density of the sediment. Such an approximation is only reasonable when the sliding proceeds in a quasi-static process. Otherwise, the hydraulic effects from the seawater have to be taken into account. A representative example rests with the phenomena in submarine landslides that a layer of water intrudes under the sediment and results in a lubrication effect and a decrease in the resistance between the sediment and the seabed [10,11]. This mechanism, termed as hydroplaning, is deemed a reason for unexpectedly long travel distance of submarine landslide, and its prediction obviously necessitates a fully coupled analysis of the seawater-soil interaction. Apart from that, the rheological feature of the sediment was ignored in [7-9]. A remarkable contribution in this regard lies in [12] where the Storegga Slide was simulated using a two-phase flow model. The interaction between the seawater and the sediment was coupled in the framework of Computational Fluid Dynamics (CFD) that a Newtonian flow model was applied for representing seawater and a non-Newtonian flow model for the rheological behaviour of sediments. The solid behaviour of the sediment was somewhat accounted for through deducing the threshold yield stress with plastic strains.

Indeed, the seawater-soil (or fluid-solid) coupled analysis is a challenge in the simulation of submarine landslides. According to the solution scheme, the numerical approaches for a fluid-solid interaction problem may be broadly categorised into the monolithic approach and the partitioned approach. The monolithic approach attempts to remould the entire problem (e.g. fluids and solids) into a single system equation that can be resolved via a unified algorithm $[13,14]$. The fluid and the solid in such a manner are thus coupled implicitly with the interfacial conditions

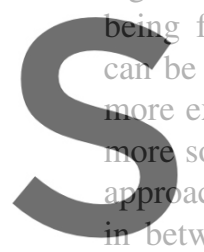
fulfilled naturally within
achieved via this couplin
expertise. For the submarin
sophisticated soil models at
ach $[15,16]$, on the other h
ween is achieved through
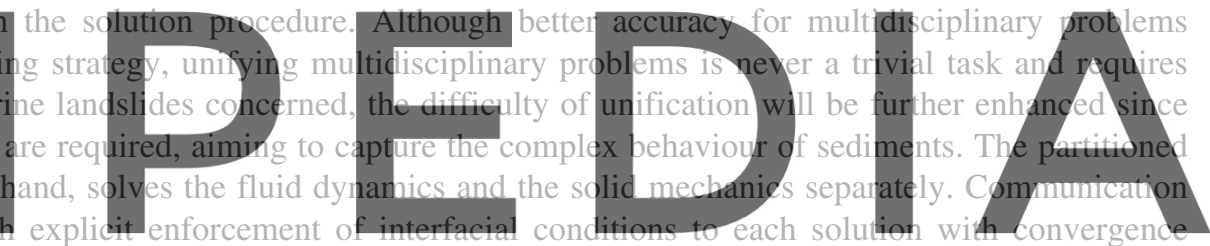

being expected via iteration loops. An apparent advantage of the partitioned approach is its capability of handling

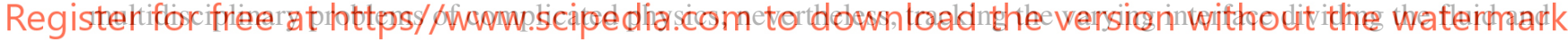
solid domains, which is not known a priori, is burdensome

In this paper, we propose a computational framework that couples fluids and solids monolithically and is capable of modelling submarine landslides and their consequences. In the framework, the formulations for solids and fluids are unified based on a mixed variational principle-the generalised Hellinger-Reissner variational principle. The relevant finite element equations for solids and fluids are reformulated into an equivalent optimisation problem, for example the second-order cone programming (SOCP) problem. The resulting optimisation problems for fluids and solids are exactly of the same form and possess the same basic variables. Thus, they can be solved by a unique solution algorithm regardless of whether the concerned problems are Newtonian/Non-Newtonian flows or elastic/elastoviscoplastic solids. This makes the coupling of nonlinear solids with fluids realised naturally which is in contrast to the available monolithic fluid/solid solvers that are usually limited to only consider the interaction between fluids and elastic solids/structures [17-20]. Additionally, the resulting finite element problem is solved in mathematical programming (MP) using a standard optimisation algorithm (e.g. the primal-dual interior point method), which differs from the available monolithic fluid/solid solvers that adopt the nested solution algorithm based on the traditional Newton-Raphson iteration [17-20]. An apparent advantage of this solution strategy rests with the fact that its convergence property can be discussed and analysed mathematically. For instance, the strong global and local convergence properties of the primal-dual interior point method (which is used in this study) for nonlinear programming have been proven in [21]. Mathematical analysis of the stability and convergence rates of the primal-dual interior point method for semidefinite programming, to which the SOCP can be converted, has also been conducted [22]. Another advantage of the proposed MP-based finite element solution scheme is the straightforward treatment of singularities in some yield criteria, for example, the Mohr-Coulomb model for a solid and the Bingham model for a fluid. Indeed, the Mohr-Coulomb model and the Bingham model have to be rounded in the nested finite element method $[23,24]$, whereas they are expressed as standard cone constraints $[25,26]$ and treated naturally in 
the SOCP. Furthermore, the extension from single-surface plasticity to multi-surface plasticity in the SOCP causes no problems and no additional computational effort which has been shown in [25]. To tackle issues resulting from extreme deformation such as mesh distortion and free-surface evolution, the final monolithically coupled formulation is merged into the Particle Finite Element Method. The proposed approach is verified against numerous benchmarks and its possibility for modelling the entire process of a submarine landslide from failure triggering through transportation to deposition in a single seamless simulation is demonstrated. Its capability in the evaluation of the direct impact of a submarine landslide on offshore infrastructure such as gas pipelines and the indirect impact via generating a tsunami is also shown.

The paper is organised as follows. Section 2 presents the standard formulation for the second-order cone programming (SOCP) problem that the finite element formulation for solids/fluids will be remoulded into. The procedures for the reformulation of the discretised governing equations for fluids and solids into an optimisation problem are then presented in Sections 3 and 4, respectively. Section 5 details the scheme for coupling the solid and the fluid using the mixed finite element and Section 6 briefly introduces the particle finite element method. Numerical examples are given in Section 7 for demonstrating the correctness and robustness of the proposed approach before conclusions are drawn in Section 8.

\section{Second-order cone programming}

Second-order cone programming (SOCP), also referred as conic quadratic optimisation, is a generalisation of linear and quadratic programming that allows the variables to be constrained inside second-order cones. When there are no linear inequality constraints, a standard SOCP program involves an optimisation problem of the form
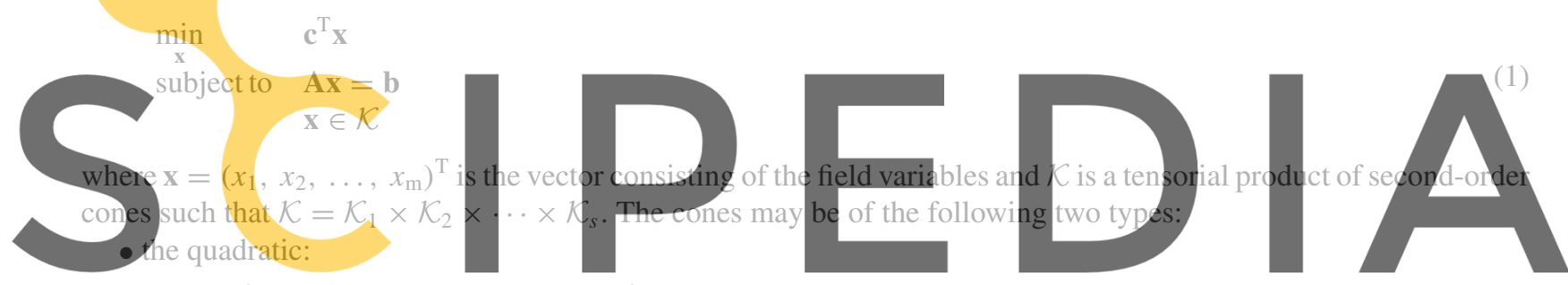

Register for free at https $\mathcal{K}_{q}=\left\{x \in \mathbb{R}^{\mathrm{m}} \mid x_{1} \geq \sqrt{x_{2}^{2}+\cdots+x_{\mathrm{m}}^{2}}\right\}$ dia.com to download the version without the watermark or

- the rotated quadratic:

$$
\mathcal{K}_{r}=\left\{x \in \mathbb{R}^{\mathrm{m}} \mid 2 x_{1} x_{2} \geq x_{3}^{2}+\cdots+x_{\mathrm{m}}^{2}, x_{1}, x_{2} \geq 0\right\}
$$

Numerous problems in solid mechanics have so far been remoulded as a SOCP problem. Typical examples include computational limit analysis of solids and plates [27-29], static/dynamic analysis of elastoplastic/elastoviscoplastic frames and solids [25,30,31], deformation and consolidation analysis of porous media [32], particle dynamic simulations (e.g. discrete element method or granular contact dynamics) [33-35], and fracture in brittle rocks [36] and jointed rock [37] among others.

Comparing to the contributions in the solid realm, mathematical programming solution techniques for fluids are much fewer. Most efforts were devoted to the so-called augmented Lagrangian approach $[38,39]$ and its accelerated variant [40] for non-Newtonian flows. They are developed based on the variational inequalities [41] and serve as an alternative to the regularised model (e.g. the Herschel-Bulkley model which replaces the non-smooth viscoplastic constitutive law by a smooth purely viscous mode) to solve viscoplastic fluid flows. Recently, Bleyer et al. $[26,42]$ reformulated the governing equations for steady yield flows as an equivalent SOCP problem which was then resolved using the primal-dual interior point method. It was shown in $[26,42]$ that the SOCP programming is much more efficient and the issue related to the singularity in the non-Newtonian flow is circumvented.

This paper first reformulates the non-steady Newtonian/Non-Newtonian flow as a SOCP problem. Rather than adopting the minimum principle as in [26,42], this study makes use of the generalised Hellinger-Reissner variational principle for the reformulation so that the resulting optimisation problems for fluids and solids are unified. It will be shown later that the final optimisation problems for fluids and solids not only are of the same form but also possess the same basic variables, which makes their monolithic coupling fulfilled smoothly. 


\section{Mathematical programming formulation of Newtonian/Non-Newtonian fluids}

This section aims to reformulate the governing equations of Newtonian and Non-Newtonian fluids into a standard optimisation problem.

\subsection{Governing equations}

We herein first consider the Bingham flow which is a typical non-Newtonian model. In case of incompressibility, the governing equations (with Einstein's notations) are as follows according to [26]:

$$
\begin{aligned}
& \sigma_{i j, j}+b_{i}=\rho \ddot{u}_{i} \\
& \dot{u}_{i, i}=0 \\
& \dot{\varepsilon}_{i j}=\frac{1}{2}\left(\dot{u}_{i, j}+\dot{u}_{j, i}\right)
\end{aligned}
$$

where $\sigma_{i j}$ is the stress tensor, $\varepsilon_{i j}{ }_{i j}$ is the strain rate tensor, $b_{i}$ is the volume body force, $\rho$ is the density of the fluid, $u_{i}$ is the displacement with a superposed dot representing differentiation with respect to time, $s_{i j}=\operatorname{dev}\left(\sigma_{i j}\right)=\sigma_{i j}-\frac{1}{3} \sigma_{k k}$ $\delta_{i}$ is the deviatoric stress tensor. Equations in (7) are the constitutive model for a Bingham flow distinguishing a

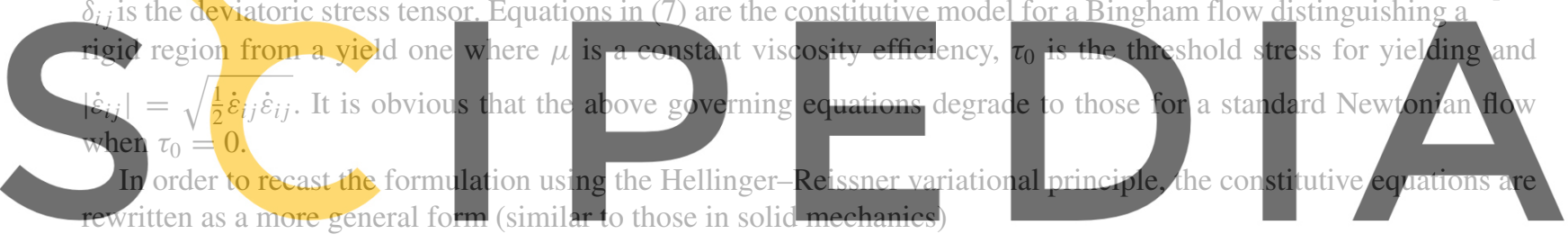

Register for frée at htitips//www.scipedia.com to download the version without the waternark

$$
\begin{aligned}
& \dot{\varepsilon}_{i j}=\dot{\lambda} \frac{\partial F\left(\tau_{i j}\right)}{\partial \tau_{i j}} \\
& \dot{\lambda} F\left(\tau_{i j}\right)=0 ; \dot{\lambda} \geq 0 ; F\left(\tau_{i j}\right) \leq 0
\end{aligned}
$$

where $\dot{\lambda}$ is the rate of the non-negative plastic multiplier, $F$ in this case is the Von Mises yield function (e.g. $F\left(\sigma_{i j}\right)=$ $\left.\sqrt{\frac{1}{2} s_{i j} s_{i j}}-\tau_{0}\right), \tau_{i j}$ is the stress lying on the boundary of $F\left(\right.$ e.g. $\left.F\left(\tau_{i j}\right)=0\right)$ and the quantity $\sigma_{i j}-\tau_{i j}$ is called the overstress which is null when $F\left(\sigma_{i j}\right) \leq 0$.

To prove the equivalence between the set of constraints (8)-(10) and the constitutive model in (7), condition (9) is first expressed as

$$
\dot{\varepsilon}_{i j}=\dot{\lambda} \frac{\operatorname{dev}\left(\tau_{i j}\right)}{2 \tau_{0}}
$$

via the substitution of the following relations

$$
\begin{aligned}
& F\left(\tau_{i j}\right)=\sqrt{\frac{1}{2} \operatorname{dev}\left(\tau_{i j}\right) \operatorname{dev}\left(\tau_{i j}\right)}-\tau_{0}=0 \\
& \frac{\partial F\left(\tau_{i j}\right)}{\partial \tau_{i j}}=\frac{\operatorname{dev}\left(\tau_{i j}\right)}{\sqrt{2 \operatorname{dev}\left(\tau_{i j}\right) \operatorname{dev}\left(\tau_{i j}\right)}}
\end{aligned}
$$

For the von Mises criterion, the incompressible condition $\dot{\varepsilon}_{k k}=0$ always holds and meanwhile Eq. (8) may be rewritten as

$$
2 \mu \dot{\varepsilon}_{i j}=s_{i j}-\operatorname{dev}\left(\tau_{i j}\right)
$$


The deviatoric part of $\tau_{i j}$ is proportional to the rate of shear strain tensor $\dot{\varepsilon}_{i j}$, namely

$$
\frac{\operatorname{dev}\left(\tau_{i j}\right)}{\left|\operatorname{dev}\left(\tau_{i j}\right)\right|}=\frac{\dot{\varepsilon}_{i j}}{\left|\dot{\varepsilon}_{i j}\right|}
$$

Because $\tau_{i j}$ is located on the yield surface that $F\left(\tau_{i j}\right)=0$, we have $\left|\operatorname{dev}\left(\tau_{i j}\right)\right|=\tau_{0}$. Thus, Eq. (15) can then be expressed as

$$
\operatorname{dev}\left(\tau_{i j}\right)=\tau_{0} \frac{\dot{\varepsilon}_{i j}}{\left|\dot{\varepsilon}_{i j}\right|} \quad \text { if } F\left(\sigma_{i j}\right)>0
$$

Substituting Eq. (16) into Eq. (14) renders

$$
2 \mu \dot{\varepsilon}_{i j}=s_{i j}-\tau_{0} \frac{\dot{\varepsilon}_{i j}}{\left|\dot{\varepsilon}_{i j}\right|} \quad \text { if } F\left(\sigma_{i j}\right)>0
$$

which is the second constraint in (7). When $F\left(\sigma_{i j}\right)<0$ is fulfilled (which also means $F\left(\tau_{i j}\right)<0$ since $\sigma_{i j}=\tau_{i j}$ in this case), constraints in (10) indicate a null plastic strain, that is also the total strain in this case, which is in line with the first constraint in (7). Thus the set of Eqs. (8)-(10) is equivalent to the constitutive model in (7). Using vector-matrix notations, the governing equations for a Bingham flow can now be expressed in a more general form of

$$
\begin{aligned}
& \nabla^{\mathrm{T}} \boldsymbol{\sigma}+\mathbf{b}=\rho \ddot{\mathbf{u}} \\
& \dot{\boldsymbol{\varepsilon}}=\nabla^{\mathrm{T}} \dot{\boldsymbol{u}} \\
& \boldsymbol{\sigma}=\tau+2 \mu \dot{\varepsilon}
\end{aligned}
$$
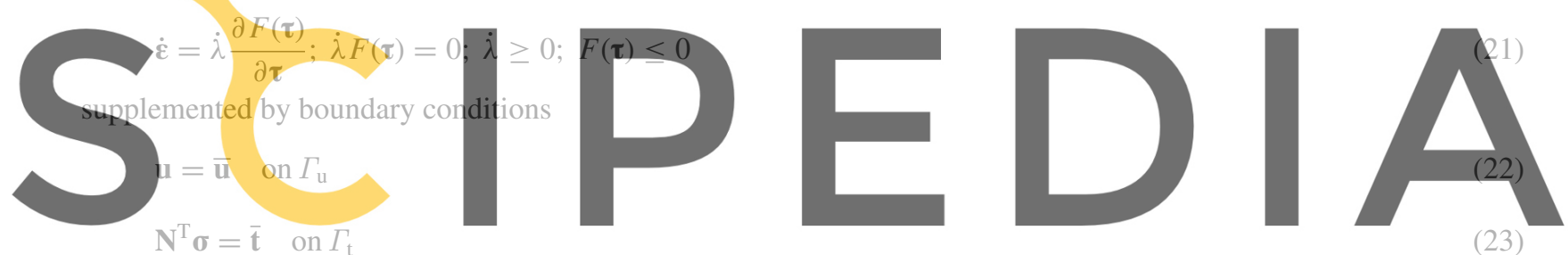

(23)

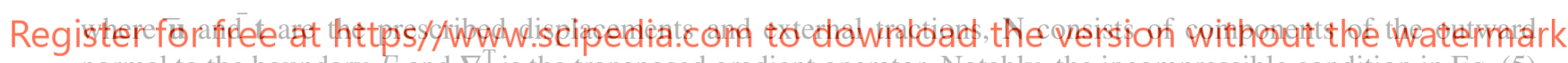
normal to the boundary $\Gamma_{\mathrm{t}}$ and $\nabla^{\mathrm{T}}$ is the transposed gradient operator. Notably, the incompressible condition in Eq. (5) does not need to be included explicitly since the utilisation of Von Mises model implies null volumetric change.

\subsection{Time discretisation}

Since a direct-time integration approach will be used for dynamic analysis, the governing Eqs. (18)-(23) have to be discretised before the equivalent variational principle is proposed. Using the standard $\theta$-method, the momentum conservation Eq. (18) and the natural boundary condition (23) are discretised in time as:

$$
\begin{aligned}
& \nabla^{\mathrm{T}}\left[\theta_{1} \boldsymbol{\sigma}_{\mathrm{n}+1}+\left(1-\theta_{1}\right) \boldsymbol{\sigma}_{\mathrm{n}}\right]+\mathbf{b}=\rho \frac{\mathbf{v}_{\mathrm{n}+1}-\mathbf{v}_{\mathrm{n}}}{\Delta t} \\
& \theta_{2} \mathbf{v}_{\mathrm{n}+1}+\left(1-\theta_{2}\right) \mathbf{v}_{\mathrm{n}}=\frac{\mathbf{u}_{\mathrm{n}+1}-\mathbf{u}_{\mathrm{n}}}{\Delta t} \\
& \mathbf{N}^{\mathrm{T}}\left(\theta_{1} \boldsymbol{\sigma}_{\mathrm{n}+1}+\left(1-\theta_{1}\right) \boldsymbol{\sigma}_{\mathrm{n}}\right)=\overline{\mathbf{t}}_{\mathrm{n}+1} \quad \text { on } \Gamma_{\mathrm{t}}
\end{aligned}
$$

where $\mathbf{v}$ are velocities, $\theta_{1}$ and $\theta_{2}$ are parameters taking values in [0,1], the subscripts $\mathrm{n}$ and $\mathrm{n}+1$ refer to the known and new, unknown states, and $\Delta t=t_{\mathrm{n}+1}-t_{\mathrm{n}}$ is the time step. Rearranging the above equations leads to

$$
\begin{aligned}
& \nabla^{\mathrm{T}} \boldsymbol{\sigma}_{\mathrm{n}+1}+\frac{1-\theta_{1}}{\theta_{1}} \nabla^{\mathrm{T}} \boldsymbol{\sigma}_{\mathrm{n}}+\widetilde{\mathbf{b}}=\widetilde{\rho} \frac{\Delta \mathbf{u}}{\Delta t^{2}} \\
& \mathbf{v}_{\mathrm{n}+1}=\frac{1}{\theta_{2}}\left[\frac{\Delta \mathbf{u}}{\Delta t}-\left(1-\theta_{2}\right) \mathbf{v}_{\mathrm{n}}\right] \\
& \mathbf{N}^{\mathrm{T}}\left(\boldsymbol{\sigma}_{\mathrm{n}+1}+\frac{1-\theta_{1}}{\theta_{1}} \boldsymbol{\sigma}_{\mathrm{n}}\right)=\tilde{\mathbf{t}} \quad \text { on } \Gamma_{\mathrm{t}} \quad \text { with } \tilde{\mathbf{t}}=\frac{1}{\theta_{1}} \overline{\mathbf{t}}_{\mathrm{n}+1}
\end{aligned}
$$


where $\Delta \mathbf{u}=\mathbf{u}_{\mathrm{n}+1}-\mathbf{u}_{\mathrm{n}}$ are the displacement increments and

$$
\widetilde{\mathbf{b}}=\frac{1}{\theta_{1}} \mathbf{b}+\widetilde{\rho} \frac{\mathbf{v}_{\mathrm{n}}}{\Delta t} \quad \text { with } \tilde{\rho}=\frac{\rho}{\theta_{1} \theta_{2}}
$$

The essential boundary condition is

$$
\mathbf{u}_{\mathrm{n}+1}=\overline{\mathbf{u}}_{\mathrm{n}+1} \quad \text { on } \Gamma_{\mathrm{u}}
$$

The constitutive equations of the Bingham model can also be discretised by introducing a parameter $\theta_{3} \in[0,1]$ :

$$
\begin{aligned}
& \left(\boldsymbol{\sigma}_{\mathrm{n}}+\theta_{3} \Delta \boldsymbol{\sigma}\right)-\left(\boldsymbol{\tau}_{\mathrm{n}}+\theta_{3} \Delta \boldsymbol{\tau}\right)=\mu \frac{\Delta \boldsymbol{\varepsilon}}{\Delta t} \Rightarrow(\Delta \boldsymbol{\sigma}-\Delta \boldsymbol{\tau})+\frac{1}{\theta_{3}}\left(\boldsymbol{\sigma}_{\mathrm{n}}-\boldsymbol{\tau}_{\mathrm{n}}\right)=\frac{\mu}{\theta_{3} \Delta t} \Delta \boldsymbol{\varepsilon} \\
& \Delta \boldsymbol{\varepsilon}=\nabla^{\mathrm{T}}(\Delta \mathbf{u})=\Delta \lambda \nabla_{\boldsymbol{\tau}} F\left(\boldsymbol{\tau}_{\mathrm{n}+1}\right) \\
& F\left(\boldsymbol{\tau}_{\mathrm{n}+1}\right) \leq 0 ; \Delta \lambda \geq 0 ; \Delta \lambda F\left(\boldsymbol{\tau}_{\mathrm{n}+1}\right)=0
\end{aligned}
$$

In summary, the governing equations for incremental analysis of Bingham flows consist of conditions in (27), (29), and (31)-(34). The velocity at the end of each incremental analysis can be updated according to Eq. (28) explicitly. The Newtonian flow is recovered by setting the threshold stress $\tau_{0}=0$.

\subsection{Generalised Hellinger-Reissner variational principle}

A generalised Hellinger-Reissner (HR) variational principle is established in this section for the Incremental analysis of the reformulated Bingham flow problem. In HR principle, both displacements and stresses are master fields, which is in contrast to the principle of minimum potential energy in which displacements are the only master
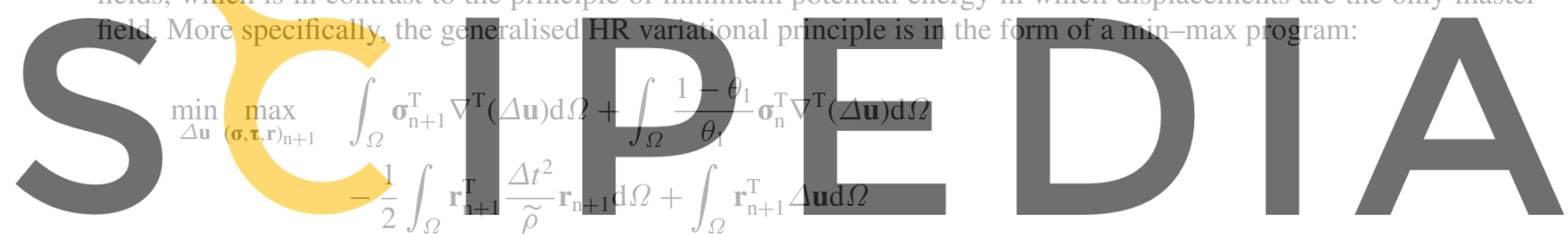

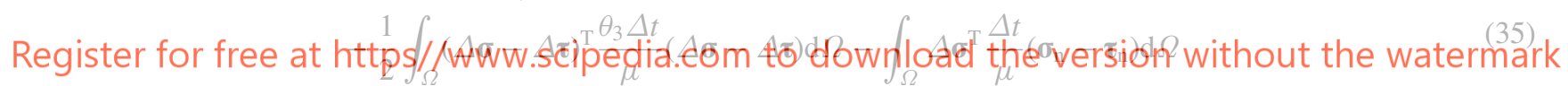

$$
+\int_{\Omega}\left(\sigma_{\mathrm{n}}-\tau_{\mathrm{n}}\right)^{\mathrm{T}} \frac{\Delta t}{\mu} \Delta \tau \mathrm{d} \Omega-\int_{\Omega} \tilde{\mathrm{b}}^{\mathrm{T}} \Delta \mathbf{u d} \Omega-\int_{\Gamma_{\mathrm{t}}} \tilde{\mathfrak{t}}^{\mathrm{T}} \Delta \mathbf{u d} \Gamma
$$

subject to $\quad F\left(\boldsymbol{\tau}_{\mathrm{n}+1}\right) \leq 0$

where $\Delta \mathbf{u}, \boldsymbol{\sigma}, \boldsymbol{\tau}$, and $\mathbf{r}$ are master fields. The physical meaning of the new variable $\mathbf{r}$ is the dynamic force that will be shown shortly.

The optimal solution of the principle (35) in fact is the solution of the discretised governing equations (e.g. (27), (29), and (31)-(34)), which can be proven as follows. Following the interior-point methodology [43], principle (35) is solved by first introducing a positively-restricted variable $s_{\mathrm{n}+1}$ so that the inequality constraint is transferred into an equality constraint

$$
\begin{aligned}
\min _{\Delta \mathbf{u}} \max _{(\boldsymbol{\sigma}, \boldsymbol{\tau}, \mathbf{r})_{\mathrm{n}+1}} & \int_{\Omega} \boldsymbol{\sigma}_{\mathrm{n}+1}^{\mathrm{T}} \nabla^{\mathrm{T}}(\Delta \mathbf{u}) \mathrm{d} \Omega+\int_{\Omega} \frac{1-\theta_{1}}{\theta_{1}} \boldsymbol{\sigma}_{\mathrm{n}}^{\mathrm{T}} \nabla^{\mathrm{T}}(\Delta \mathbf{u}) \mathrm{d} \Omega \\
& -\frac{1}{2} \int_{\Omega} \mathbf{r}_{\mathrm{n}+1}^{\mathrm{T}} \frac{\Delta t^{2}}{\widetilde{\rho}} \mathbf{r}_{\mathrm{n}+1} \mathrm{~d} \Omega+\int_{\Omega} \mathbf{r}_{\mathrm{n}+1}^{\mathrm{T}} \Delta \mathbf{u d} \Omega \\
& -\frac{1}{2} \int_{\Omega}(\Delta \boldsymbol{\sigma}-\Delta \boldsymbol{\tau})^{\mathrm{T}} \frac{\theta_{3} \Delta t}{\mu}(\Delta \boldsymbol{\sigma}-\Delta \boldsymbol{\tau}) \mathrm{d} \Omega-\int_{\Omega} \Delta \boldsymbol{\sigma}^{\mathrm{T}} \frac{\Delta t}{\mu}\left(\boldsymbol{\sigma}_{\mathrm{n}}-\boldsymbol{\tau}_{\mathrm{n}}\right) \mathrm{d} \Omega \\
& +\int_{\Omega}\left(\boldsymbol{\sigma}_{\mathrm{n}}-\boldsymbol{\tau}_{\mathrm{n}}\right)^{\mathrm{T}} \frac{\Delta t}{\mu} \Delta \boldsymbol{\tau} \mathrm{d} \Omega-\int_{\Omega} \widetilde{\mathbf{b}}^{\mathrm{T}} \Delta \mathbf{u d} \Omega-\int_{\Gamma_{\mathrm{t}}} \widetilde{\mathbf{t}}^{\mathrm{T}} \Delta \mathbf{u d} \Gamma+\int_{\Omega} \beta \ln s_{\mathrm{n}+1} \mathrm{~d} \Omega
\end{aligned}
$$

subject to

$$
F\left(\boldsymbol{\tau}_{\mathrm{n}+1}\right)+s_{\mathrm{n}+1}=0
$$


where $\beta$ is an arbitrarily small positive constant. The penalty term $\beta \ln s_{\mathrm{n}+1}$ in the objective function imposes the non-negativity requirement on $s_{\mathrm{n}+1}$, and is known as a logarithmic barrier function.

The Lagrangian associated with the optimisation problem (36) now can be expressed as

$$
\begin{aligned}
& \mathcal{L}_{f}\left(\Delta \mathbf{u}, \boldsymbol{\sigma}_{\mathrm{n}+1}, \boldsymbol{\tau}_{\mathrm{n}+1}, \mathbf{r}_{\mathrm{n}+1}, \Delta \lambda, s_{\mathrm{n}+1}\right) \\
& =\int_{\Omega} \boldsymbol{\sigma}_{\mathrm{n}+1}^{\mathrm{T}} \nabla^{\mathrm{T}}(\Delta \mathbf{u}) \mathrm{d} \Omega+\int_{\Omega} \frac{1-\theta_{1}}{\theta_{1}} \boldsymbol{\sigma}_{\mathrm{n}}^{\mathrm{T}} \nabla^{\mathrm{T}}(\Delta \mathbf{u}) \mathrm{d} \Omega-\frac{1}{2} \int_{\Omega} \mathbf{r}_{\mathrm{n}+1}^{\mathrm{T}} \frac{\Delta t^{2}}{\widetilde{\rho}} \mathbf{r}_{\mathrm{n}+1} \mathrm{~d} \Omega+\int_{\Omega} \mathbf{r}_{\mathrm{n}+1}^{\mathrm{T}} \Delta \mathbf{u d} \Omega \\
& \quad-\frac{1}{2} \int_{\Omega}(\Delta \boldsymbol{\sigma}-\Delta \boldsymbol{\tau})^{\mathrm{T}} \frac{\theta_{3} \Delta t}{\mu}(\Delta \boldsymbol{\sigma}-\Delta \boldsymbol{\tau}) \mathrm{d} \Omega-\int_{\Omega} \Delta \boldsymbol{\sigma}^{\mathrm{T}} \frac{\Delta t}{\mu}\left(\boldsymbol{\sigma}_{\mathrm{n}}-\boldsymbol{\tau}_{\mathrm{n}}\right) \mathrm{d} \Omega+\int_{\Omega}\left(\boldsymbol{\sigma}_{\mathrm{n}}-\boldsymbol{\tau}_{\mathrm{n}}\right)^{\mathrm{T}} \frac{\Delta t}{\mu} \Delta \boldsymbol{\tau} \mathrm{d} \Omega \\
& \quad-\int_{\Omega} \widetilde{\mathbf{b}}^{\mathrm{T}} \Delta \mathbf{u d} \Omega-\int_{\Gamma_{\mathrm{t}}} \widetilde{\mathbf{t}}^{\mathrm{T}} \Delta \mathbf{u d} \Gamma+\int_{\Omega} \beta \ln s_{\mathrm{n}+1} \mathrm{~d} \Omega-\int_{\Omega} \Delta \lambda\left(F\left(\boldsymbol{\tau}_{\mathrm{n}+1}\right)+s_{\mathrm{n}+1}\right) \mathrm{d} \Omega
\end{aligned}
$$

The first-order necessary and sufficient Karush-Kuhn-Tucker (KKT) optimality conditions associated with (36) can be derived by the variation of the above Lagrangian with respect to the design variables. Specifically, the associated KKT conditions are:

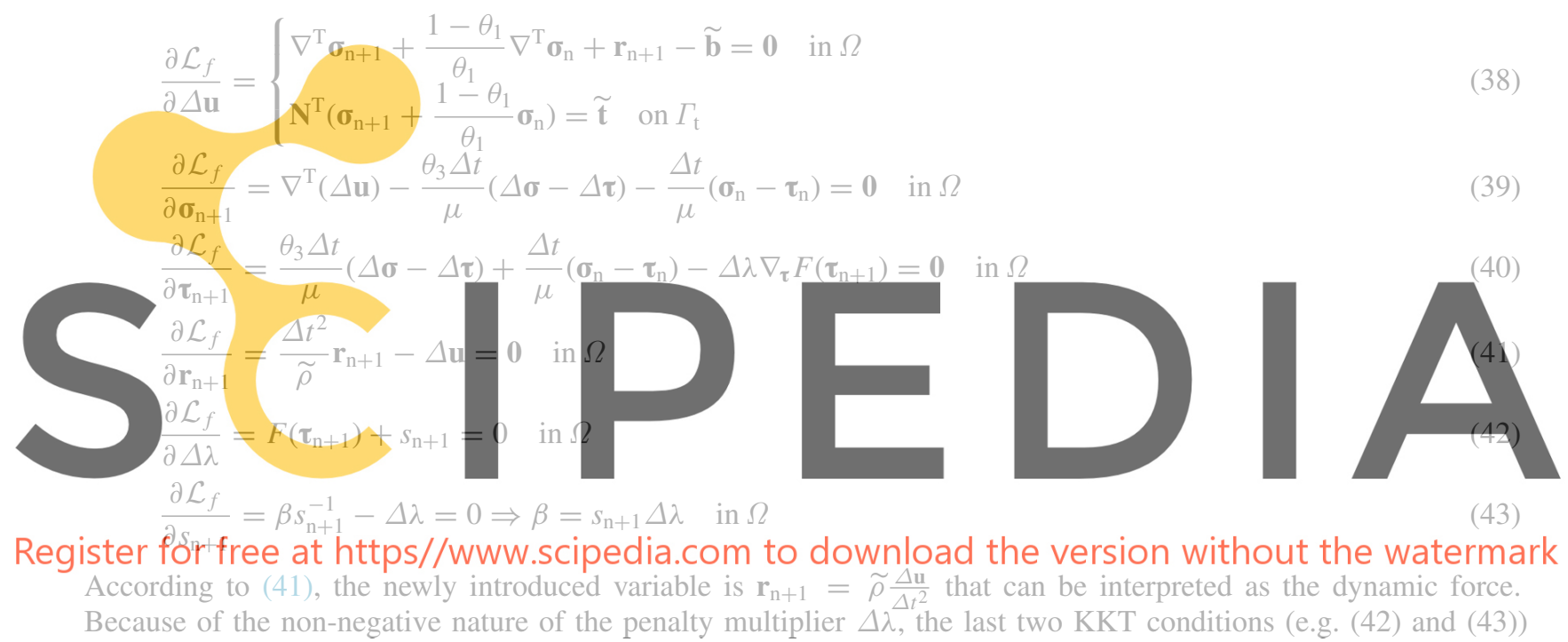

recover the yield condition and the complementarity condition shown in (34) when $\beta \rightarrow 0^{+}$. The rest of the KKT conditions (e.g. Eqs. (38)-(41)) are apparently the discretised governing equations presented in Section 3.2 (e.g. Eqs. (27), (29), (32) and (33)). In other words, the first-order necessary and sufficient KKT optimality conditions associated with the principle (36) are equivalent to the discretised governing equations; and thus the principle (36) is valid. This also implies the validity of the principle (35) for Bingham flows since the principle (36) is approaching (35) when $\beta \rightarrow 0^{+}$.

\section{Mathematical programming formulation of solid dynamics}

Since the governing equations for the non-Newtonian flow are expressed in a general form, the extension of the relevant optimisation problem to the one for an elastoviscoplastic solid is forthright. The governing equations for the dynamics of an elastoviscoplastic solid are the same as those for fluid dynamics except for the differences in the constitutive equations. The constitutive equations for an elastoviscoplastic solid are

$$
\begin{aligned}
& \boldsymbol{\sigma}=\boldsymbol{\tau}+2 \mu \dot{\boldsymbol{\varepsilon}}^{\mathrm{vp}} \\
& \dot{\boldsymbol{\varepsilon}}=\nabla^{\mathrm{T}} \dot{\mathbf{u}}=\dot{\boldsymbol{\varepsilon}}^{\mathrm{e}}+\dot{\boldsymbol{\varepsilon}}^{\mathrm{vp}} \\
& \dot{\boldsymbol{\varepsilon}}^{\mathrm{e}}=\mathbb{C} \dot{\boldsymbol{\sigma}} \\
& \dot{\boldsymbol{\varepsilon}}^{\mathrm{vp}}=\dot{\lambda} \frac{\partial F(\boldsymbol{\tau})}{\partial \boldsymbol{\tau}} ; \dot{\lambda} F(\boldsymbol{\tau})=0 ; \dot{\lambda} \geq 0
\end{aligned}
$$


which, along with the momentum balance Eq. (18) and the boundary conditions (22) and (23), compose the complete governing equations for the relevant dynamic analysis. Again, the constitutive equations are similar to those for Bingham flows except that, according to (45), the rate of the total strain rate ${ }^{\cdot} \boldsymbol{\varepsilon}$ is divided into an elastic part $\boldsymbol{\varepsilon}^{\cdot \mathrm{e}}$, that is related to the stress via the Hooke's law (46) with $\mathbb{C}$ being elastic compliance matrix, and a viscoplastic part $\boldsymbol{\varepsilon}^{\cdot \mathrm{vp}}$ calculated using the rule of plastic flow (47). This is in contrast to the case in Section 3 that any strain induced refers to unrecoverable 'plastic strain'. Thus the min-max problem (35) only needs to further include the elastic part for incremental elastoviscoplastic analysis of a solid which is

$$
\begin{aligned}
\min _{\Delta \mathbf{u}} \max _{(\boldsymbol{\sigma}, \boldsymbol{\tau}, \mathbf{r}) \mathrm{n}+1} & \frac{-\frac{1}{2} \int_{\Omega} \Delta \boldsymbol{\sigma}^{\mathrm{T}} \mathbb{C} \Delta \boldsymbol{\sigma} \mathrm{d} \Omega}{\text { Elasticity }}+\int_{\Omega} \boldsymbol{\sigma}_{\mathrm{n}+1}^{\mathrm{T}} \nabla^{\mathrm{T}}(\Delta \mathbf{u}) \mathrm{d} \Omega+\int_{\Omega} \frac{1-\theta_{1}}{\theta_{1}} \boldsymbol{\sigma}_{\mathrm{n}}^{\mathrm{T}} \nabla^{\mathrm{T}}(\Delta \mathbf{u}) \mathrm{d} \Omega \\
& -\frac{1}{2} \int_{\Omega} \mathbf{r}_{\mathrm{n}+1}^{\mathrm{T}} \frac{\Delta t^{2}}{\widetilde{\rho}} \mathbf{r}_{\mathrm{n}+1} \mathrm{~d} \Omega+\int_{\Omega} \mathbf{r}_{\mathrm{n}+1}^{\mathrm{T}} \Delta \mathbf{u d} \Omega \\
& -\frac{1}{2} \int_{\Omega}(\Delta \boldsymbol{\sigma}-\Delta \boldsymbol{\tau})^{\mathrm{T}} \frac{\theta_{3} \Delta t}{\mu}(\Delta \boldsymbol{\sigma}-\Delta \boldsymbol{\tau}) \mathrm{d} \Omega-\int_{\Omega} \Delta \boldsymbol{\sigma}^{\mathrm{T}} \frac{\Delta t}{\mu}\left(\boldsymbol{\sigma}_{\mathrm{n}}-\boldsymbol{\tau}_{\mathrm{n}}\right) \mathrm{d} \Omega \\
& +\int_{\Omega}\left(\sigma_{\mathrm{n}}-\tau_{\mathrm{n}}\right)^{\mathrm{T}} \frac{\Delta t}{\mu} \Delta \tau \mathrm{d} \Omega-\int_{\Omega} \widetilde{\mathrm{b}}^{\mathrm{T}} \Delta \mathrm{ud} \Omega-\int_{\Gamma_{\mathrm{t}}} \widetilde{t}^{\mathrm{T}} \Delta \mathrm{ud} \Gamma
\end{aligned}
$$

\section{subject to $\quad F\left(\tau_{\mathrm{n}+1}\right) \leq 0$}

The associated Lagrangian, after the transition of the inequality constraint into an equality one as carried out in the last section, is expressed as
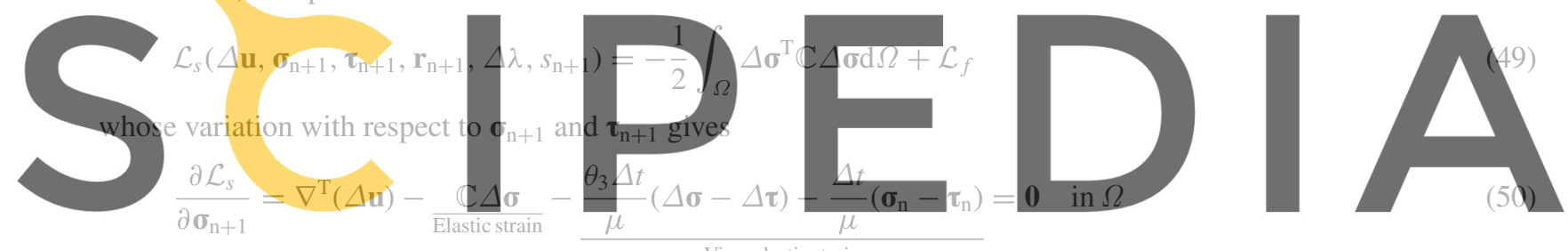

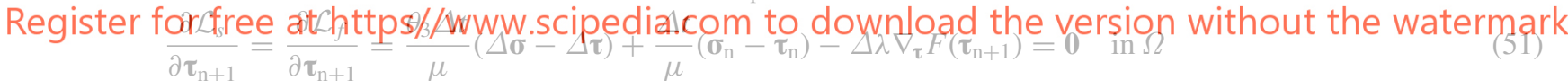

Substitution of Eq. (51) into (50) results in the addition decomposition of the total strain rate as in Eq. (45). The variation of $\mathcal{L}_{s}$ with respect to other variables (e.g. $\Delta \mathbf{u}, \mathbf{r}_{\mathrm{n}+1}, \Delta \lambda$, and $s_{\mathrm{n}+1}$ ) results in Eqs. (38), (41)-(43), which verifies the equivalence between the optimisation problem (48) and the discretised governing equations for dynamic analysis of an elastoviscoplastic solid.

Material hardening/softening behaviour can also be accounted for in the principle according to [31]. Suppose that a yield criterion function with strain hardening/softening is in the form of $F(\boldsymbol{\tau}, \kappa)=0$ where $\kappa=H\left(\boldsymbol{\varepsilon}^{v p}\right)$ is a set of internal variables relating to the viscoplastic strain. The associated principle according to [31] thus is

$$
\begin{aligned}
\min _{\Delta \mathbf{u}(\boldsymbol{\sigma}, \boldsymbol{\tau}, \mathbf{r}, \kappa)_{\mathrm{n}+1}} & -\frac{1}{2} \int_{\Omega} \Delta \boldsymbol{\sigma}^{\mathrm{T}} \mathbb{C} \Delta \boldsymbol{\sigma} \mathrm{d} \Omega+\int_{\Omega} \boldsymbol{\sigma}_{\mathrm{n}+1}^{\mathrm{T}} \nabla^{\mathrm{T}}(\Delta \mathbf{u}) \mathrm{d} \Omega+\int_{\Omega} \frac{1-\theta_{1}}{\theta_{1}} \boldsymbol{\sigma}_{\mathrm{n}}^{\mathrm{T}} \nabla^{\mathrm{T}}(\Delta \mathbf{u}) \mathrm{d} \Omega \\
& -\frac{1}{2} \int_{\Omega} \mathbf{r}_{\mathrm{n}+1}^{\mathrm{T}} \frac{\Delta t^{2}}{\widetilde{\rho}} \mathbf{r}_{\mathrm{n}+1} \mathrm{~d} \Omega+\int_{\Omega} \mathbf{r}_{\mathrm{n}+1}^{\mathrm{T}} \Delta \mathbf{u d} \Omega \\
& -\frac{1}{2} \int_{\Omega}(\Delta \boldsymbol{\sigma}-\Delta \boldsymbol{\tau})^{\mathrm{T}} \frac{\theta_{3} \Delta t}{\eta}(\Delta \boldsymbol{\sigma}-\Delta \boldsymbol{\tau}) \mathrm{d} \Omega-\int_{\Omega} \Delta \boldsymbol{\sigma}^{\mathrm{T}} \frac{\Delta t}{\eta}\left(\boldsymbol{\sigma}_{\mathrm{n}}-\boldsymbol{\tau}_{\mathrm{n}}\right) \mathrm{d} \Omega \\
& +\int_{\Omega}\left(\boldsymbol{\sigma}_{\mathrm{n}}-\boldsymbol{\tau}_{\mathrm{n}}\right)^{\mathrm{T}} \frac{\Delta t}{\eta} \Delta \boldsymbol{\tau} \mathrm{d} \Omega-\frac{1}{\frac{2}{\text { Hardening/Softening term }}} \mathbb{H}_{t}^{-1} \Delta \kappa^{2} \mathrm{~d} \Omega-\int_{\Omega} \widetilde{\mathbf{b}}^{\mathrm{T}} \Delta \mathbf{u d} \Omega-\int_{\Gamma_{\mathrm{t}}} \widetilde{\mathbf{t}}^{\mathrm{T}} \Delta \mathbf{u} \mathrm{d} \Gamma
\end{aligned}
$$

subject to

$$
F\left(\boldsymbol{\tau}_{\mathrm{n}+1}, \underline{\kappa_{\mathrm{n}+1}}\right) \leq 0
$$


where the underlined terms are newly introduced due to the hardening/softening and $\mathbb{H}_{t}$ is constitutive modulus that reads

$$
\mathbb{H}_{t}=-\frac{d H\left(\boldsymbol{\varepsilon}_{\mathrm{n}}^{\mathrm{vp}}\right)}{d \boldsymbol{\varepsilon}^{\mathrm{vp}}} \frac{\nabla_{\boldsymbol{\tau}} F\left(\boldsymbol{\tau}_{\mathrm{n}}, \kappa_{\mathrm{n}}\right)}{\nabla_{\kappa} F\left(\boldsymbol{\tau}_{\mathrm{n}}, \kappa_{\mathrm{n}}\right)}
$$

The inclusion of material hardening/softening in the principle has been detailed in [31] and thus is not further discussed in this paper.

In brief, variational principle (52) is a general optimisation problem for elastoviscoplastic analysis which degrades to principle (35)

$$
\begin{aligned}
\min _{\Delta \mathbf{u}} \max _{(\boldsymbol{\sigma}, \boldsymbol{\tau}, \mathbf{r})_{\mathrm{n}+1}} & \int_{\Omega} \boldsymbol{\sigma}_{\mathrm{n}+1}^{\mathrm{T}} \nabla^{\mathrm{T}}(\Delta \mathbf{u}) \mathrm{d} \Omega+\int_{\Omega} \frac{1-\theta_{1}}{\theta_{1}} \boldsymbol{\sigma}_{\mathrm{n}}^{\mathrm{T}} \nabla^{\mathrm{T}}(\Delta \mathbf{u}) \mathrm{d} \Omega \\
& -\frac{1}{2} \int_{\Omega} \mathbf{r}_{\mathrm{n}+1}^{\mathrm{T}} \frac{\Delta t^{2}}{\widetilde{\rho}} \mathbf{r}_{\mathrm{n}+1} \mathrm{~d} \Omega+\int_{\Omega} \mathbf{r}_{\mathrm{n}+1}^{\mathrm{T}} \Delta \mathbf{u d} \Omega \\
& -\frac{1}{2} \int_{\Omega}(\Delta \sigma-\Delta \tau)^{\mathrm{T}} \frac{\theta_{3} \Delta t}{\mu}(\Delta \sigma-\Delta \tau) \mathrm{d} \Omega-\int_{\Omega} \Delta \sigma^{\mathrm{T}} \frac{\Delta t}{\mu}\left(\sigma_{\mathrm{n}}-\tau_{\mathrm{n}}\right) \mathrm{d} \Omega \\
& +\int_{\Omega}\left(\sigma_{\mathrm{n}}-\tau_{\mathrm{n}}\right)^{\mathrm{T}} \frac{\Delta t}{\mu} \Delta \tau \mathrm{d} \Omega-\int_{\Omega} \widetilde{\mathrm{b}}^{\mathrm{T}} \Delta \mathrm{ud} \Omega-\int_{\Gamma_{\mathrm{t}}} \tilde{t}^{\mathrm{T}} \Delta \mathbf{u d} \Gamma \\
\text { subject to } \quad & F\left(\tau_{\mathrm{n}+1}\right) \leq 0
\end{aligned}
$$

for the incremental analysis of Newtonian/Non-Newtonian flows when the parts relevant to the elasticity and material hardening/softening are erased. When the Von Mises yield criterion is used, the above problem is for analysing the standard Bingham flow. While the

Moreover, principle (52) degrades to

related to viscosity that is
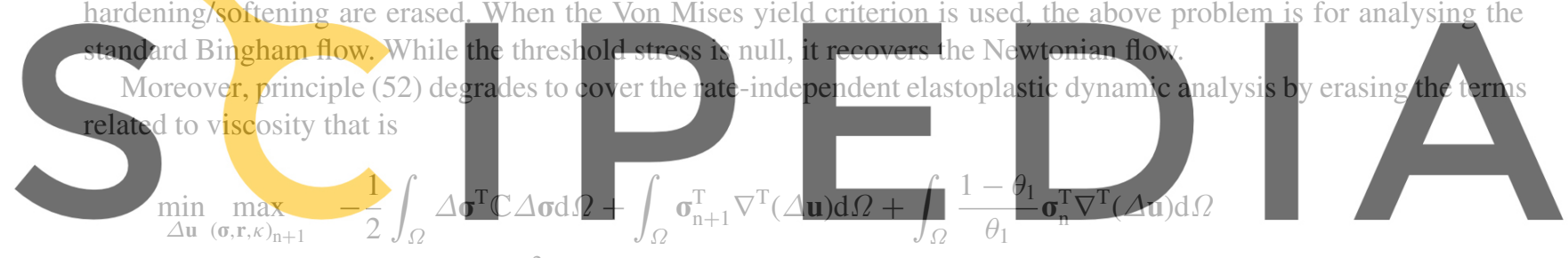

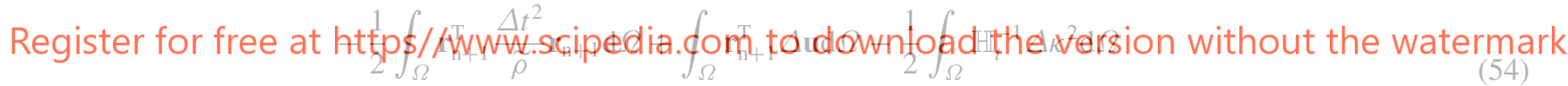

$$
-\int_{\Omega} \widetilde{\mathbf{b}}^{\mathrm{T}} \Delta \mathrm{u} \mathrm{d} \Omega-\int_{\Gamma_{\mathrm{t}}} \tilde{\mathbf{t}}^{\mathrm{T}} \Delta \mathrm{ud} \Gamma
$$

subject to $F\left(\boldsymbol{\sigma}_{\mathrm{n}+1}, \kappa_{\mathrm{n}+1}\right) \leq 0$

and to cover the elastoplastic static analysis [25] by further erasing the dynamic terms that is

$$
\begin{aligned}
\min _{\Delta \mathbf{u}(\boldsymbol{\sigma}, \kappa)} \max _{\mathrm{n}+1} & -\frac{1}{2} \int_{\Omega} \Delta \boldsymbol{\sigma}^{\mathrm{T}} \mathbb{C} \Delta \boldsymbol{\sigma} \mathrm{d} \Omega+\int_{\Omega} \boldsymbol{\sigma}_{\mathrm{n}+1}^{\mathrm{T}} \nabla^{\mathrm{T}}(\Delta \mathbf{u}) \mathrm{d} \Omega-\frac{1}{2} \int_{\Omega} \mathbb{H}_{t}^{-1} \Delta \kappa^{2} \mathrm{~d} \Omega \\
& -\int_{\Omega} \mathbf{b}^{\mathrm{T}} \Delta \mathbf{u d} \Omega-\int_{\Gamma_{\mathrm{t}}} \mathbf{t}_{\mathrm{n}+1}^{\mathrm{T}} \Delta \mathbf{u d} \Gamma
\end{aligned}
$$

subject to $F\left(\boldsymbol{\sigma}_{\mathrm{n}+1}, \kappa_{\mathrm{n}+1}\right) \leq 0$

The upper bound limit analysis $[25,28]$ is also recovered by removing the elastic part and hardening/softening part, which is

$$
\begin{array}{ll}
\min _{\Delta \mathbf{u}(\boldsymbol{\sigma}, \alpha)} & \int_{\Omega} \boldsymbol{\sigma}^{\mathrm{T}} \nabla^{\mathrm{T}}(\Delta \mathbf{u}) \mathrm{d} \Omega-\int_{\Omega} \mathbf{b}^{\mathrm{T}} \Delta \mathbf{u d} \Omega-\alpha \int_{\Gamma_{\mathrm{t}}} \mathbf{t}^{\mathrm{T}} \Delta \mathbf{u} \mathrm{d} \Gamma \\
\text { subject to } & F(\boldsymbol{\sigma}) \leq 0
\end{array}
$$

where $\alpha$ is a new introduced variable representing the factor of the imposed traction force. Notably, all the above problems refer to total stress analysis. This is because the marine clay is commonly simulated in undrained conditions [7,9] according to its low permeability. Nevertheless, the analysis of saturated porous media can also 


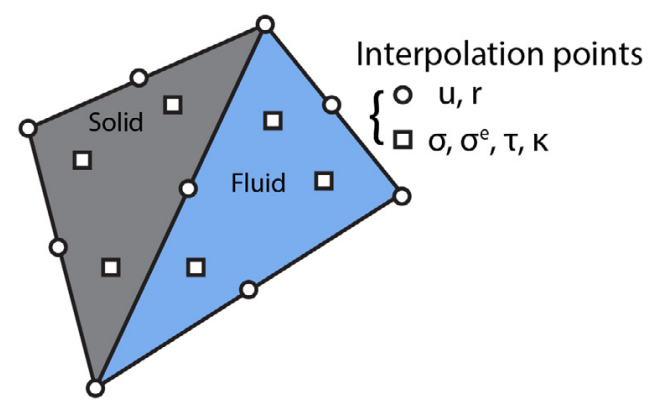

Fig. 2. The mixed isoparametric triangular element in use and the corresponding interpolation points for different master fields.

be cast into the same form which has been discussed in [32] where the effective stress and pore water pressure are the master fields.

\section{Monolithic coupling and solution technique}

The min-max problem (52) is first discretised using the standard finite element shape function owing to generalised feature, and then the coupling between the fluid and the solid is discussed. As both the displacementlike and stress-like fields are master fields in the generalised HR variational principle, they have to be interpolated by shape functions independently such as

$$
\begin{aligned}
& \boldsymbol{\sigma}(\mathbf{x}) \approx \mathbf{N}_{\boldsymbol{\sigma}} \hat{\boldsymbol{\sigma}}, \quad \boldsymbol{\sigma}^{\mathrm{e}}(\mathbf{x}) \approx \mathbf{N}_{\mathbf{\sigma}^{\mathrm{e}}} \hat{\boldsymbol{\sigma}}^{\mathrm{e}}, \boldsymbol{\tau}(\mathbf{x}) \approx \mathbf{N}_{\mathbf{\tau}} \hat{\boldsymbol{\tau}}, \\
& \mathbf{r}(\mathbf{x}) \approx \mathbf{N}_{\mathbf{r}} \hat{\mathbf{r}}, \quad \mathbf{u}(\mathbf{x}) \approx \mathbf{N}_{\mathbf{u}} \hat{\mathbf{u}}, \quad \nabla^{\mathrm{T}} \mathbf{u} \approx \mathbf{B}_{\mathbf{u}} \hat{\mathbf{u}}, \\
& \kappa(\mathbf{x}) \approx \mathbf{N}_{\kappa} \hat{\boldsymbol{\kappa}}
\end{aligned}
$$

where $\hat{\boldsymbol{\sigma}}, \hat{\boldsymbol{\sigma}}^{\mathrm{e}}, \hat{\boldsymbol{\tau}}, \hat{\mathbf{r}}, \hat{\mathbf{u}}$, and $\hat{\boldsymbol{\kappa}}$ are vectors containing the values of the corresponding field variables at interpolation points, $\mathbf{N}$ is a matrix consisting of shape functions, and $\mathbf{B}_{\mathrm{u}}=\nabla^{\mathrm{T}} \mathbf{N}_{\mathrm{u}}$. Since the mixed variational principle is used, the field variables shown in (57) are all independent variables. The mixed isoparametric triangular element shown in Fig. 2 is used for the approximation of both the solid and the fluid. The master fields of displacement $\mathbf{u}$ and dynamic force $\hat{\mathbf{r}}$ are interpolated based on the vertex and the mid-side nodes of the triangle (e.g. the circles in Fig. 2), whereas the master fields of stress-like states $\hat{\boldsymbol{\sigma}}, \hat{\boldsymbol{\sigma}}^{\wedge}, \hat{\boldsymbol{\tau}}, \hat{\boldsymbol{\kappa}}$ are interpolated based on the internal points (e.g. the squares in Fig. 2) with the area coordinates $\beta_{j}$ being $\left(\beta_{j-1}, \beta_{j}, \beta_{j+1}\right)=\left({ }_{6}^{1}, \frac{4}{6}, \frac{1}{6}\right), j=1,2,3$. In other words, the master fields of the displacement and the dynamic force use the same quadratic shape function, and master fields of the stress-like states use the same linear shape function. We refer the reader to [25] for more details of mixed elements of this kind where their property and performance were discussed.

By substituting Eq. (57), the principle (e.g. (52)) discretised in space reads

$$
\begin{aligned}
\min _{\Delta \hat{\mathbf{u}}\left(\hat{\boldsymbol{\sigma}}, \hat{\boldsymbol{\tau}}, \hat{\boldsymbol{\sigma}}^{\mathrm{e}} \hat{\mathbf{r}}, \hat{\mathbf{\kappa}}\right)_{\mathrm{n}+1}} & -\frac{1}{2} \Delta \hat{\boldsymbol{\sigma}}^{\mathrm{T}} \mathbf{C} \Delta \hat{\boldsymbol{\sigma}}+\Delta \hat{\mathbf{u}}^{\mathrm{T}} \mathbf{B}^{\mathrm{T}} \hat{\boldsymbol{\sigma}}_{\mathrm{n}+1}+\Delta \hat{\mathbf{u}}^{\mathrm{T}} \frac{1-\theta_{1}}{\theta_{1}} \mathbf{B}^{\mathrm{T}} \hat{\boldsymbol{\sigma}}_{\mathrm{n}} \\
& -\frac{1}{2} \hat{\mathbf{r}}_{\mathrm{n}+1}^{\mathrm{T}} \mathbf{D} \hat{\mathbf{r}}_{\mathrm{n}+1}+\Delta \hat{\mathbf{u}}^{\mathrm{T}} \mathbf{A}^{\mathrm{T}} \hat{\mathbf{r}}_{\mathrm{n}+1}-\frac{1}{2} \Delta \hat{\boldsymbol{\sigma}}^{\mathrm{e} \mathrm{T}} \mathbf{M} \Delta \hat{\boldsymbol{\sigma}}^{\mathrm{e}} \\
& -\Delta \hat{\boldsymbol{\sigma}}^{\mathrm{e} \mathrm{T}} \mathbf{f}^{\mathrm{c}}-\frac{1}{2} \Delta \hat{\boldsymbol{\kappa}}^{\mathrm{T}} \mathbf{H} \Delta \hat{\boldsymbol{\kappa}}-\Delta \hat{\mathbf{u}}^{\mathrm{T}} \mathbf{f}^{\mathrm{e}}
\end{aligned}
$$

subject to

$$
\begin{aligned}
& \Delta \hat{\boldsymbol{\sigma}}^{\mathrm{e}}=\Delta \hat{\boldsymbol{\sigma}}-\Delta \hat{\boldsymbol{\tau}} \\
& F_{j}\left(\hat{\boldsymbol{\tau}}_{\mathrm{n}+1}, \hat{\boldsymbol{\kappa}}_{\mathrm{n}+1}\right) \leq 0, j=1,2, \ldots, N_{G}
\end{aligned}
$$

where an intermediate variable $\boldsymbol{\sigma}^{\mathrm{e}}=\boldsymbol{\sigma}-\boldsymbol{\tau}$ termed the overstress is introduced, $N_{G}$ is the total number of integration points for instance Gauss points, and

$$
\begin{aligned}
& \mathbf{C}=\int_{\Omega} \mathbf{N}_{\boldsymbol{\sigma}}^{\mathrm{T}} \mathbb{C} \mathbf{N}_{\boldsymbol{\sigma}} \mathrm{d} \Omega, \quad \mathbf{B}^{\mathrm{T}}=\int_{\Omega} \mathbf{B}_{\mathbf{u}}^{\mathrm{T}} \mathbf{N}_{\boldsymbol{\sigma}} \mathrm{d} \Omega, \\
& \mathbf{D}=\int_{\Omega} \mathbf{N}_{\mathbf{r}}^{\mathrm{T}} \frac{\Delta t^{2}}{\widetilde{\rho}} \mathbf{N}_{\mathbf{r}} \mathrm{d} \Omega, \quad \mathbf{A}^{\mathrm{T}}=\int_{\Omega} \mathbf{N}_{\mathbf{u}}^{\mathrm{T}} \mathbf{N}_{\mathbf{r}} \mathrm{d} \Omega,
\end{aligned}
$$




$$
\begin{aligned}
& \mathbf{M}=\int_{\Omega} \mathbf{N}_{\mathbf{\sigma}^{\mathrm{e}}}^{\mathrm{T}} \frac{\theta_{3} \Delta t}{\eta} \mathbf{N}_{\mathbf{\sigma}^{\mathrm{e}}} \mathrm{d} \Omega, \quad \mathbf{H}=\int_{\Omega} \mathbf{N}_{\kappa}^{\mathrm{T}} \frac{1}{\mathcal{H}_{t}} \mathbf{N}_{\kappa} \mathrm{d} \Omega, \\
& \mathbf{f}^{\mathrm{e}}=\int_{\Omega} \mathbf{N}_{\mathbf{u}}^{\mathrm{T}} \widetilde{\mathbf{b}} \mathrm{d} \Omega+\int_{\Gamma_{\mathrm{t}}} \mathbf{N}_{\mathbf{u}}^{\mathrm{T}} \widetilde{\mathbf{t}} \mathrm{d} \Gamma, \mathbf{f}^{\mathbf{c}}=\int_{\Omega} \mathbf{N}_{\mathbf{\sigma}}^{\mathrm{T}} \frac{\Delta t}{\eta} \boldsymbol{\sigma}_{\mathrm{n}}^{\mathrm{e}} \mathrm{d} \Omega
\end{aligned}
$$

The minimisation part of principle (58) with respect to the incremental displacement $\Delta$ 'u can be resolved analytically resulting in a maximisation problem which can also be expressed as a minimisation problem with an opposite sign

$$
\begin{array}{cl}
\min _{\left(\hat{\boldsymbol{\sigma}}, \hat{\boldsymbol{\tau}}, \hat{\boldsymbol{\sigma}}^{\mathrm{e}}, \hat{\mathbf{r}}, \hat{\mathbf{\kappa}}\right)_{\mathrm{n}+1}} & \frac{1}{2} \Delta \hat{\boldsymbol{\sigma}}^{\mathrm{T}} \mathbf{C} \Delta \hat{\boldsymbol{\sigma}}+\frac{1}{2} \hat{\mathbf{r}}_{\mathrm{n}+1}^{\mathrm{T}} \mathbf{D} \hat{\mathbf{r}}_{\mathrm{n}+1}+\frac{1}{2} \Delta \hat{\boldsymbol{\sigma}}^{\mathrm{e} \mathrm{T}} \mathbf{M} \Delta \hat{\boldsymbol{\sigma}}^{\mathrm{e}} \\
& +\frac{1}{2} \Delta \hat{\boldsymbol{\kappa}}^{\mathrm{T}} \mathbf{H} \Delta \hat{\boldsymbol{\kappa}}+\Delta \hat{\boldsymbol{\sigma}}^{\mathrm{eT}} \mathbf{f}^{\mathrm{c}} \\
\text { subject to } & \mathbf{B}^{\mathrm{T}} \hat{\boldsymbol{\sigma}}_{\mathrm{n}+1}+\frac{1-\theta_{1}}{\theta_{1}} \mathbf{B}^{\mathrm{T}} \hat{\boldsymbol{\sigma}}_{\mathrm{n}}+\mathbf{A}^{\mathrm{T}} \hat{\mathbf{r}}_{\mathrm{n}+1}-\mathbf{f}^{\mathrm{e}}=\mathbf{0} \\
& \Delta \hat{\boldsymbol{\sigma}}^{\mathrm{e}}=\Delta \hat{\boldsymbol{\sigma}}-\Delta \hat{\boldsymbol{\tau}} \\
& F_{j}\left(\hat{\boldsymbol{\tau}}_{\mathrm{n}+1}, \hat{\boldsymbol{\kappa}}_{\mathrm{n}+1}\right) \leq 0, j=1,2, \ldots, N_{G}
\end{array}
$$

The finite element discretised principle for Newtonian/Non-Newtonian flow can be derived following the same way which is

$$
\begin{array}{ll}
\min _{\left(\hat{\boldsymbol{\sigma}}, \hat{\boldsymbol{\tau}}, \hat{\boldsymbol{\sigma}}^{\mathrm{e}}, \hat{\mathbf{r}}\right)_{\mathrm{n}+1}} & \frac{1}{2} \hat{\mathbf{r}}_{\mathrm{n}+1}^{\mathrm{T}} \mathbf{D} \hat{\mathbf{r}}_{\mathrm{n}+1}+\frac{1}{2} \Delta \hat{\boldsymbol{\sigma}}^{\mathrm{e} \mathrm{T}} \mathbf{M} \Delta \hat{\boldsymbol{\sigma}}^{\mathrm{e}}+\Delta \hat{\boldsymbol{\sigma}}^{\mathrm{e}} \mathbf{f}^{\mathrm{c}} \\
\text { subject to } & \mathbf{B}^{\mathrm{T}} \hat{\boldsymbol{\sigma}}_{\mathrm{n}+1}+\frac{1-\theta_{1}}{\theta_{1}} \mathbf{B}^{\mathrm{T}} \hat{\boldsymbol{\sigma}}_{\mathrm{n}}+\mathbf{A}^{\mathrm{T}} \hat{\mathbf{r}}_{\mathrm{n}+1}-\mathbf{f}^{\mathrm{e}}=\mathbf{0} \\
& \Delta \hat{\boldsymbol{\sigma}}^{\mathrm{e}}=\Delta \hat{\boldsymbol{\sigma}}-\Delta \hat{\boldsymbol{\tau}} \\
& F_{j}\left(\hat{\boldsymbol{\tau}}_{\mathrm{n}+1}\right) \leq 0, j=1,2, \ldots, N_{G}
\end{array}
$$

It is apparent that the principle (61) (for fluids) is the degradation of the principle (60) (for solids).

For the sake of convenience, the principle (60) is the one solved for both fluids and solids. When the element represents a fluid, the elastic compliance matrix $\mathbf{C}$, the constitutive modulus matrix $\mathbf{H}$ and the internal variable for softening/hardening $\hat{\boldsymbol{\kappa}} \wedge$ in principle (60) are set to be null at the corresponding elements. Such an operation simplifies the monolithic coupling of the fluid and the solid.

The transformation of the optimisation problem (60) into a standard second-order cone programming problem in the form of (1) is detailed in Appendix, and the optimisation engine MOSEK, in which the primal-dual interior point method is available, is adopted as the solver.

\section{The particle finite element method (PFEM)}

The unified formulation presented in the previous section is for the incremental finite element analysis at each single time step. When a large deformation problem is concerned, the proposed formulation encounters the issues such as mesh distortion and severe free-surface evolution due to its Lagrangian feature. To overcome the issues resulting from large change in geometry, the proposed formulation is implemented into the framework of the Particle Finite Element Method (PFEM) [44]. Consequently, it is capable of modelling submarine landslides and their consequences in which extreme material deformation is inevitable.

The PFEM makes use of the Lagrangian finite element approach (in our cases the proposed unified formulation) to solve the discretised governing equations on meshes. At the time point that meshes have a certain degree of distortion, mesh topologies are erased leaving behind mesh nodes treated as free particles. A new computational domain is then identified using the so-called $\alpha$-shape method [45] on the basis of the position of free particles followed by the remeshing of the identified domain. State variables, including those at both mesh nodes and Gauss points, are then mapped from old meshes to new meshes followed by a new incremental finite element analysis.

The variable mapping is performed using the unique element method (UEM) [46] in this study which is composed of three basic steps as follows: (i) update the old mesh according to the cumulative displacement; (ii) find which old finite element the new Gauss point (or the new mesh node) lies in; and (iii) interpolate the variable states at the new Gauss points (or the new mesh node) on the basis of the corresponding state variables at the detected old 


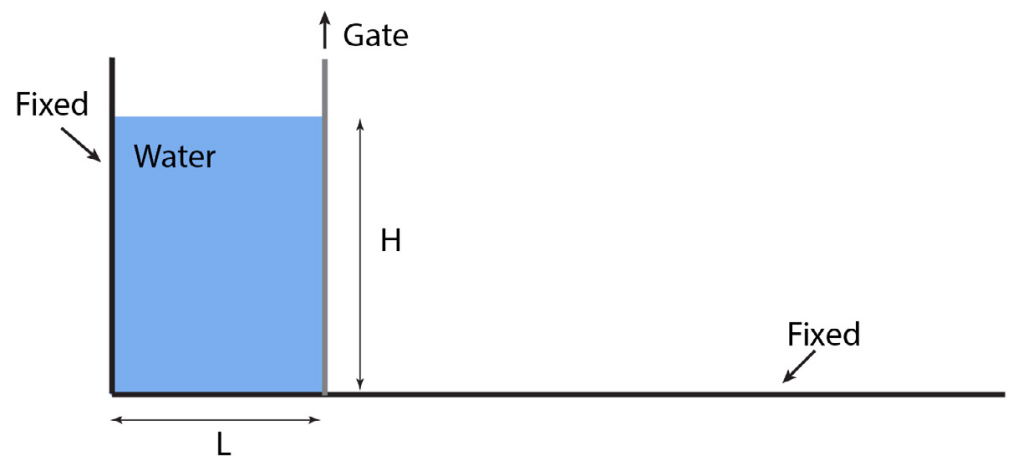

Fig. 3. Schematic illustration of water dam break.

element. The accuracy of the UEM has been estimated in detail in [46] showing that the fluctuation induced in the load-displacement curve using the UEM for bearing capacity problem is within $6 \%$ even when rather coarse meshes are used. The fluctuation can be further reduced by adopting finer meshes. It is remarkable that, in the PFEM, meshes of sufficiently small size have to be used for correct boundary identification. Previous studies [31,47] showed that the mesh of the size performs well for correct boundary identification in the PFEM also guarantees the accuracy of the UEM for variable mapping. Thus influence of variable mapping is very limited in the PFEM and converged solutions can be obtained.

To date, the PFEM has tackled numerous challenging problems such as the modelling of multi-phase flows [48], fluid-structure interactions [49,50], granular flows [47,51-53], flow of fresh cement suspensions [54], penetration problems [53,55,56], landslides [57,58] and the generated waves [59], among others.

\section{Numerical examples}

The correctness and robustness of the proposed unified solid/fluid finite element formulation (60) is verified via simulating numerous benchmarks. First, single-phase problems such as the water dam break, the annular viscometer problem, and the collapse of aluminium bars are simulated in order to verify it for modelling Newtonian flows, Non-Newtonian flows, and solid dynamics, respectively. Comparisons of our simulation results against experimental data, analytical solutions, and also results using other numerical approaches available in the literature are carried out. The efficiency of the proposed monolithic coupling for simulating multi-phase problems is then tested against an experimental test concerning the underwater granular collapse and the induced waves. Last but not least, the possibility of the approach for modelling submarine landslides and their consequences is shown by considering a model test in which the failure and the post-failure processes of an underwater slope are predicted via a single simulation with both the direct impact on infrastructure such as pipelines and the indirect impact via the generated-tsunami being estimated. In all simulations, the parameters for time discretisation are $\theta_{1}=\theta_{2}=1$ and $\theta_{3}={ }^{1}$, and the high-performance optimisation engine MOSEK [60] is used for solutions. The default values for error tolerances in MOSEK are used including the parameter $\beta$ shown in Section 3.3.

\subsection{Single-phase problems}

\subsubsection{Newtonian flow}

The first example concerned is the water dam break. The dam is initially $10 \mathrm{~cm}$ wide and $20 \mathrm{~cm}$ high as shown in Fig. 3, and the water of density $\rho=1 \times 10^{3} \mathrm{~kg} / \mathrm{m}^{3}$ is incompressible. The gravitational acceleration is $\mathrm{g}=-9.8 \mathrm{~m} / \mathrm{s}^{2}$. The lift up of the gate leads to the spreading of the water dam. As it is modelled as a Newtonian flow, the Von-Mises model is used with the cohesion (or called threshold stress in the field of fluid dynamics) being null. The domain is discretised using 3879 triangular elements with typical element size $\mathrm{h}=0.4 \mathrm{~cm}$ (e.g. the length of element edges). The time step utilised is $\Delta t=1 \times 10^{-3} \mathrm{~s}$.

The configurations of the dam-break wave at four different time instants are plotted in Fig. 4 with the distribution of water pressure being shown. Simulation results from [61] and [62], in which the Smooth Particle Hydrodynamics 

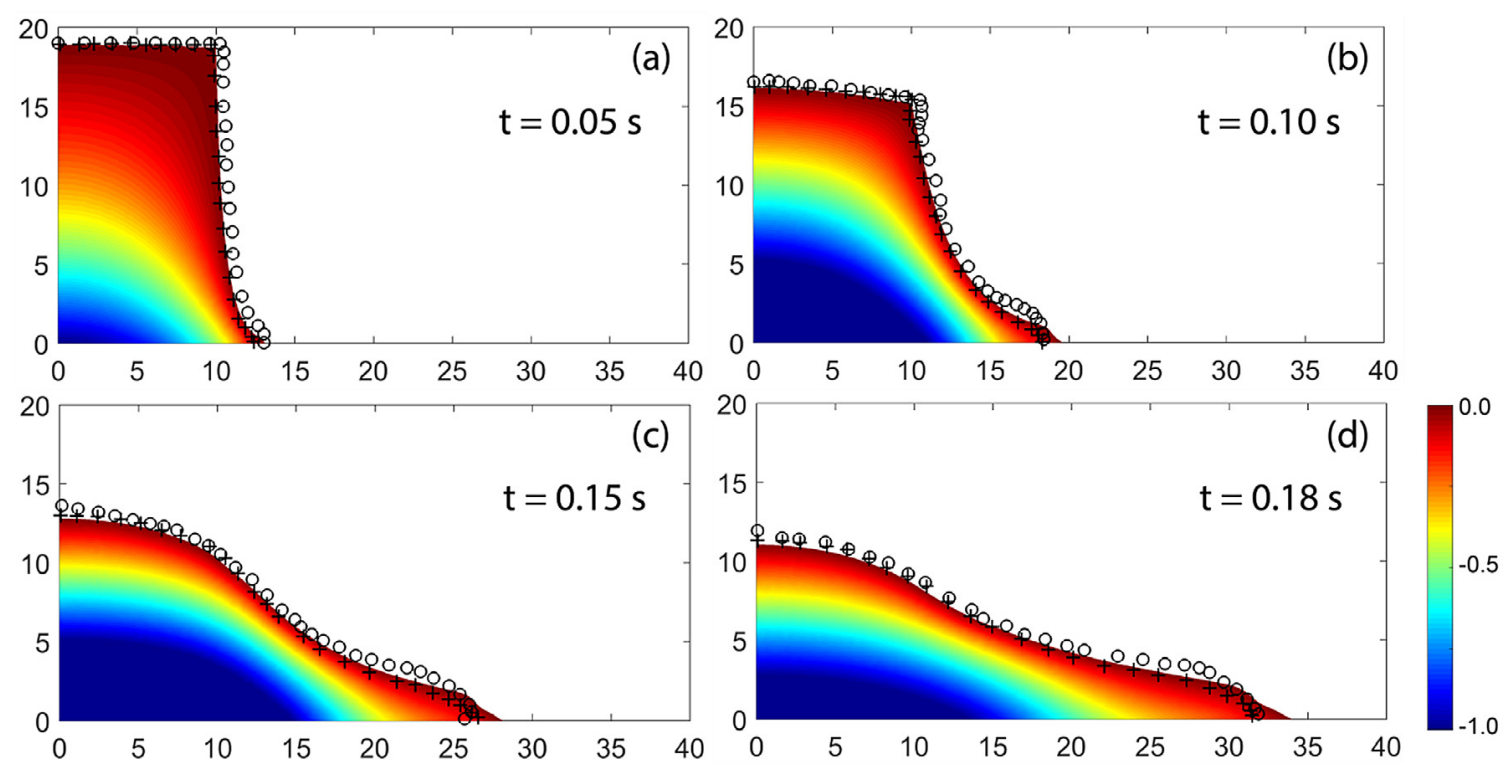

Fig. 4. Configurations of the dam-break wave with the distribution of water pressure (unit: $k P a)$ at time instances (a) $t=0.05 \mathrm{~s}$, (b) $t=0.10 \mathrm{~s}$, (c) $t=0.15 \mathrm{~s}$, and $(\mathrm{d}) \mathrm{t}=0.18 \mathrm{~s}$, respectively. Circles (o) represent the free surface obtained in [61], and crosses $(+)$ refer to that obtained in [62].

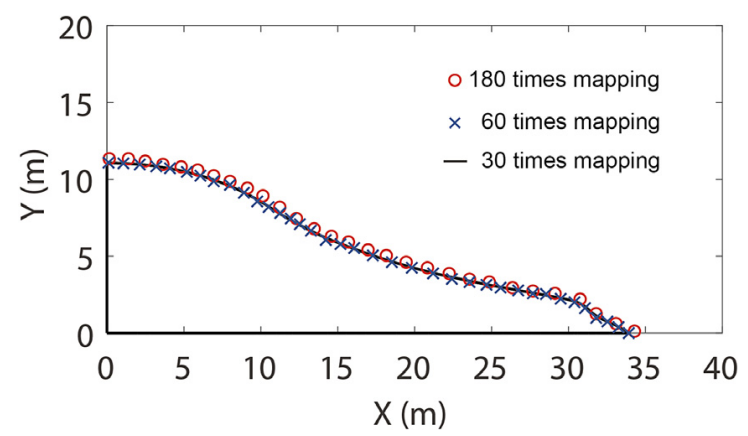

Fig. 5. Configurations of the dam-break wave at $t=0.18 \mathrm{~s}$ from the PFEM simulation with boundary identification and variable mapping conducted per 1 step (180 times), per 3 steps (60 times), and per 6 steps (30 times).

approach was used, are also illustrated for comparison purposes in Fig. 4. It is shown that the results agree with each other very well which verifies the proposed unified formulation for Newtonian flows.

Furthermore, the problem is simulated using the PFEM with boundary identification being carried out per 1 step, per 3 steps and per 6 steps. This is to estimate the influence of the operation of the variable mapping on the simulation results since variable mapping has to be carried out when boundary identification is performed. As seen in Fig. 5 that the simulation results agree well with each other. Indeed, the mesh size that is small enough to identify boundaries usually guarantees the accuracy of the UEM for variable mapping whose influence is thus very limited.

It is notable that the simulation does not suffer from volumetric locking because of the used mixed elements that the displacement field is interpolated using quadratic shape functions and the stress field is approximated linearly.

\subsubsection{Non-Newtonian flow}

The Bingham flow in an annular viscometer is investigated in order to validate the unified formulation for modelling Non-Newtonian flows in this section. The annular viscometer is made of two coaxial cylinders as shown in Fig. 6 . The outer cylinder is fixed whereas the inner cylinder rotates at a constant angular velocity $\omega$. Supposing the fluid is stick to the apparatus boundaries, analytical solutions are available which depend on the rheological properties of the 


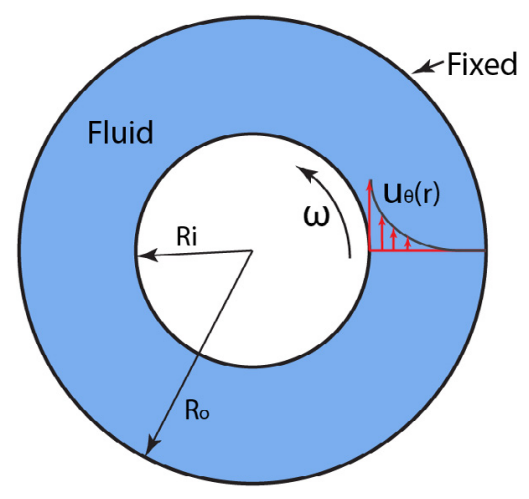

Fig. 6. A schematic illustration of an annular viscometer.

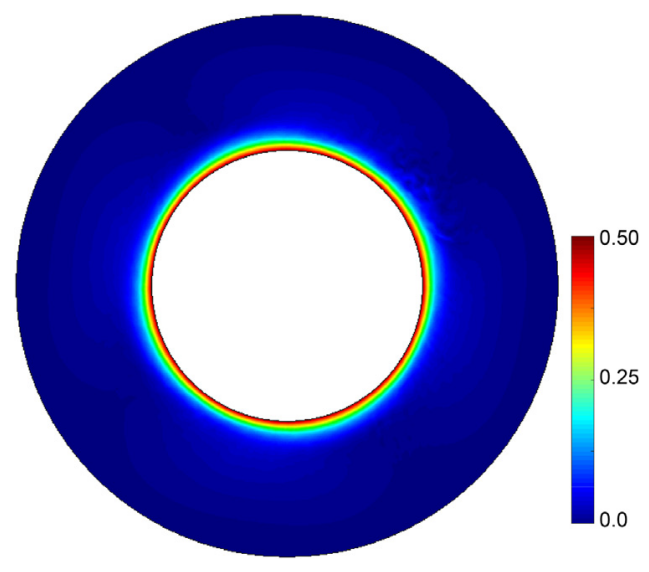

Fig. 7. Distribution of the tangential speed at the steady state (Unit: $\mathrm{m} / \mathrm{s})$.

fluid. For the considered Bingham fluid, a transition radius $R_{t}$ exists that distinguishes the sheared fluids that are close to the inner cylinder from those located in an un-yield/rigid zone. According to [63], the transition radius $R_{\mathrm{t}}$ is the solution of

$$
\left(\frac{R_{\mathrm{t}}}{R_{\mathrm{i}}}\right)^{2}-2 \ln \left(\frac{R_{\mathrm{t}}}{R_{\mathrm{i}}}\right)-\left(\frac{2 \sqrt{2} \mu \omega}{\tau_{0}}+1\right)=0
$$

and, in the sheared zone, the tangential velocity of the fluid is

$$
u_{\theta}(r)=r \frac{\sqrt{2} \tau_{0}}{\mu}\left(\left(\frac{R_{\mathrm{t}}}{r}\right)^{2}-2 \ln \left(\frac{R_{\mathrm{t}}}{r}\right)-1\right) .
$$

In this work, the radii of the outer and inner cylinders are $R_{\mathrm{O}}=100 \mathrm{~cm}$ and $R_{\mathrm{i}}=50 \mathrm{~cm}$, respectively. The viscosity fluid is $\mu=1 \mathrm{~Pa}$ s and the threshold stress $\tau_{0}=10 \mathrm{~Pa}$. The density is $\rho=1000 \mathrm{~kg} / \mathrm{m}^{3}$. The inner cylinder rotates at an angular speed of $\omega=1 \mathrm{rad} / \mathrm{s}$. The domain is discretised using meshes with a characteristic size $\mathrm{h}=3.5 \mathrm{~cm}$, and the time step for the simulation is $\Delta t=1 \times 10^{-3} \mathrm{~s}$.

Fig. 7 shows the distribution of the speed at the steady state from our simulations. As expected, the tangential speed decreases with the radial position. Note that, although this is a fluid dynamics problem in a fixed domain, issues related to sever mesh distortion still exist because the Lagrangian description/mesh is used. The corresponding tangential speed at the steady state is plotted in Fig. 8. It is shown that the transition radius obtained from the simulation is around $0.7 \mathrm{~m}$ which coincides with the analytical solution. Furthermore, the overall tangential speed at the steady 


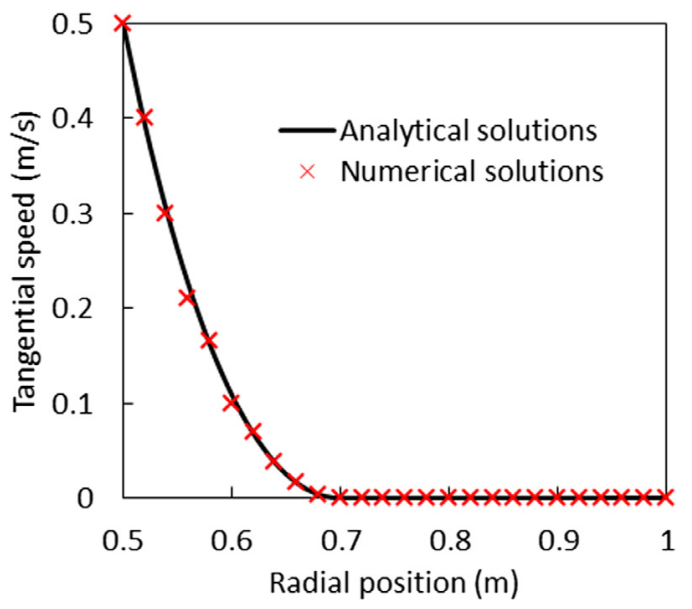

Fig. 8. Curves of the tangential speed against the radial position.

state from the simulation agrees well with the analytical solution, indicating the correctness of the proposed unified formulation for Non-Newtonian flows.

\subsubsection{Solid mechanics problem}

The third example for the single-phase problem is an experiment test of a collapse problem conducted in [64] which is similar to the water break problem. The column of the size $200 \times 100 \mathrm{~mm}$ however was composed of small aluminium bars of diameters 1 and $1.5 \mathrm{~mm}$ and length $50 \mathrm{~mm}$. This example was used to verify the SPH approach for simulating elastoplastic problems in plane strain conditions in geomechanics in [64].

In our simulations, the Mohr-Coulomb model is used to represent the material with parameters being the same as those from [64]: Young's Modulus $E=0.84 \mathrm{MPa}$, Poisson's ratio $v=0.3$, friction angle $\phi=19.8^{\circ}$, dilation angle $\psi=0^{\circ}$ and cohesion $c=0$. The density of the material is $\rho=1.8 \times 10^{3} \mathrm{~kg} / \mathrm{m}^{3}$. The viscosity of the material is neglected in this case. Simulations are carried out using a time step $\Delta t=1 \times 10^{-3} \mathrm{~s}$.

Snapshots of configurations of the column at different time instances from our simulations are shown in Fig. 9. The particles shown in the figure are mesh nodes marked in different colours. The lifting of the gate leads to an immediate collapse of the column. The top surface of the column is being eroded continuously throughout the collapse process whereas an undisturbed zone exists at the bottom left. The final profile as well as the surface of the undisturbed zone from our simulations are compared to the experimental date [64]. As seen, a great agreement is achieved verifying the proposed unified model for solid dynamics.

\subsection{Multi-phase problem}

The fourth example considered is a model test of submarine landslides and their hydraulic effects carried out by Rzadkiewicz et al. [65]. The setup is illustrated in Fig. 10. As shown, the model test consists of a triangular mass of sands that slide along an inclined surface of $45^{\circ}$ in a water channel. The sand mass is initially positioned $0.1 \mathrm{~m}$ below the water surface and its width is the same as that of the channel. The problem thus can be regarded plane-strain. This problem is commonly used for the validity of numerical approaches for multi-phase flows. In this study, it is used to verify the monolithic coupling of the proposed unified formulation for simulating multi-phase problem, in particular in terms of the water wave generated by submarine landslides.

In our simulation, the sand mass is approximated as a non-Newtonian fluid (e.g. Bingham flow) according to [65]. The material parameters used in our simulations are exactly the same as those for the case in [65] (e.g. the case with rheology but without artificial diffusivity). Specifically, the water has a density of $1000 \mathrm{~kg} / \mathrm{m}^{3}$ with both viscosity and yield stress being null. The mean density of saturated sands is $1985 \mathrm{~kg} / \mathrm{m}^{3}$ and the threshold stress is $200 \mathrm{~Pa}$. The viscosity of saturated sands is null according to [65]. The characteristic mesh size used is $\mathrm{h}=0.015 \mathrm{~m}$ and the time step is $\Delta t=1 \times 10^{-3} \mathrm{~s}$. 


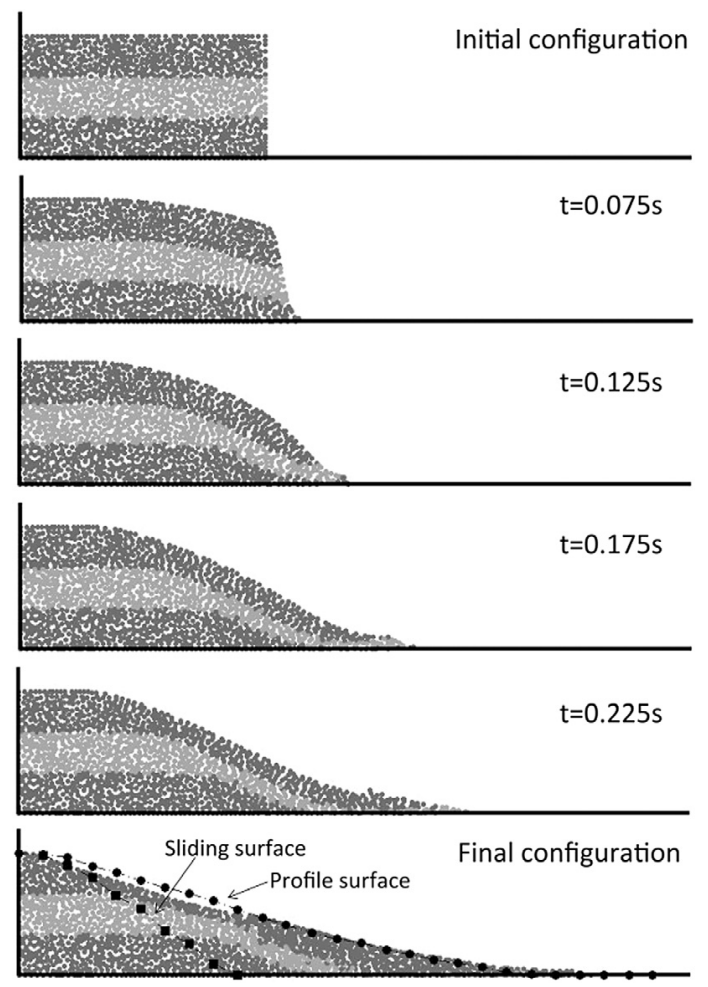

Fig. 9. Snapshots of profiles at different time instances. The sliding surface and the profile surface are experimental data from [64].

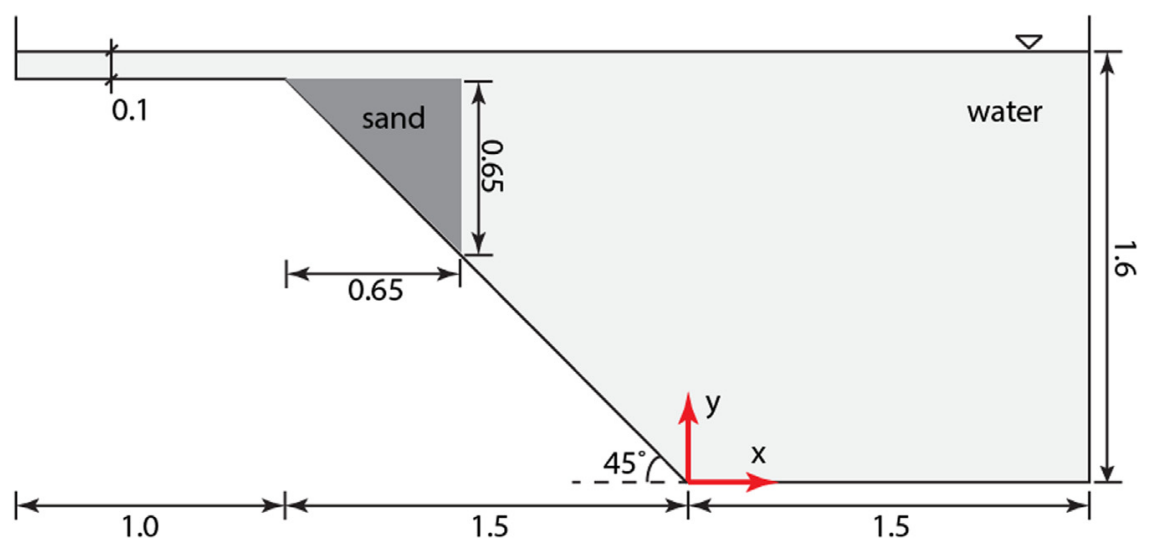

Fig. 10. A schematic illustration of the experimental test for underwater granular flows (Unit of length: $\mathrm{m}$ ).

Fig. 11 shows the snapshots of configurations of the sliding sand as well as the induced water wave at time instances of $\mathrm{t}=0.4 \mathrm{~s}$ and $0.8 \mathrm{~s}$, in which the corresponding shapes of deformed sand mass from the simulations in [65] are also shown for comparison. As shown, our simulated results agree well with those computed from [65]. It is also notable that, at $\mathrm{t}=0.8 \mathrm{~s}$, a part of sands separate from the major sliding mass (see the zoom-in image in Fig. 11(b)) and is surrounded by water, which has been captured successfully by the proposed method. Fig. 12 shows the quantitative comparison between the elevations of the free surface among our present simulation results, the computed results and the experimental data provided in [65] at those two time instances. Again, our simulations results coincide with the computed results from [65], both of which are close to the experimental data [65]. Such agreements verify the monolithic coupling of the proposed unified formulation for multi-phase problems. 

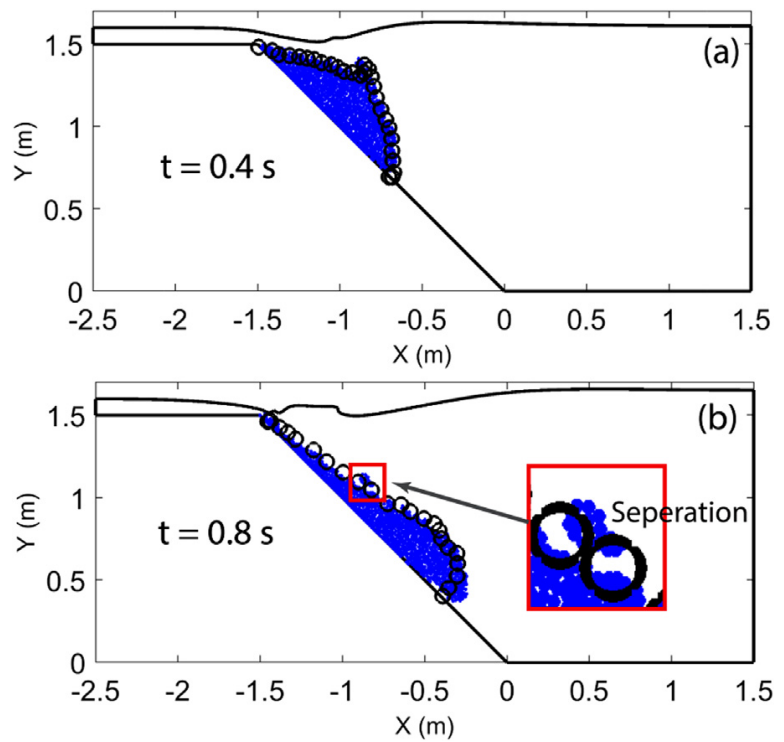

Fig. 11. Snapshots of configurations of the sand mass and the induced water wave at time instance (a) $t=0.4 \mathrm{~s}$ and (b) $\mathrm{t}=0.8 \mathrm{~s}$. Circles are computed results from [65].
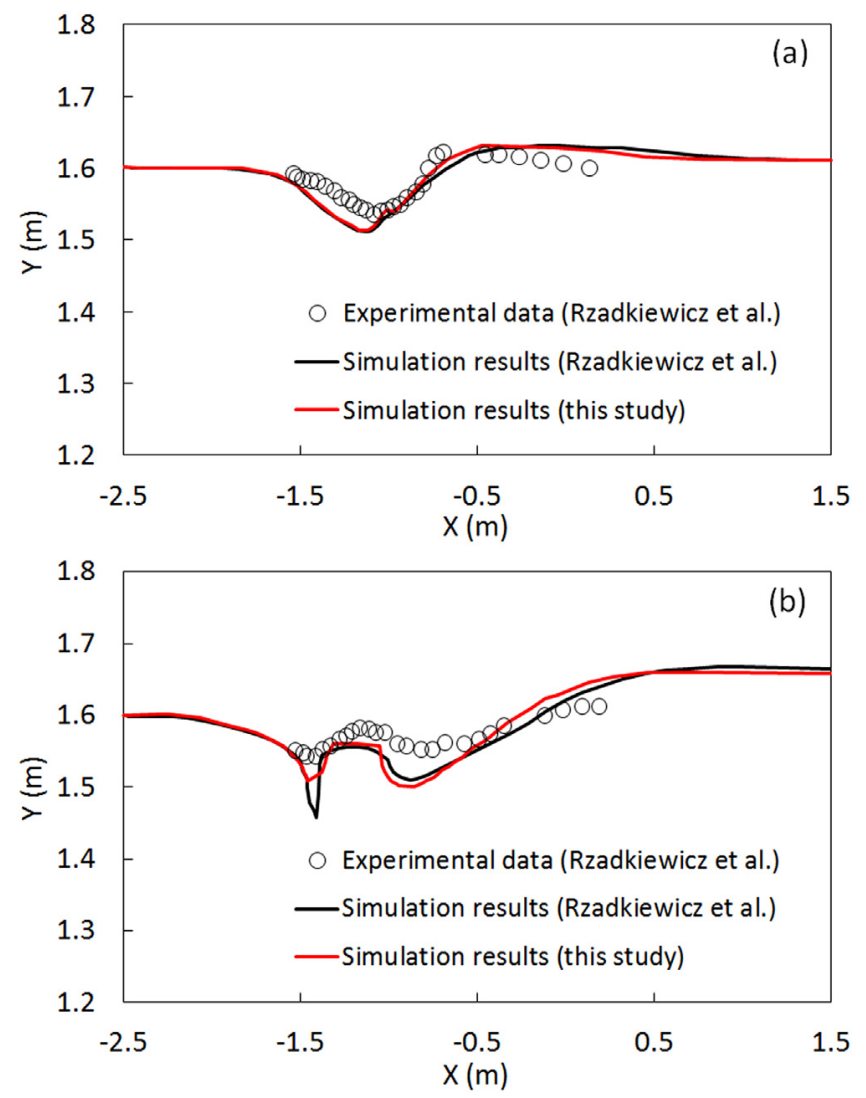

Fig. 12. Comparison of the elevations of the free surface at times (a) $t=0.4 \mathrm{~s}$ and (b) $t=0.8 \mathrm{~s}$. 


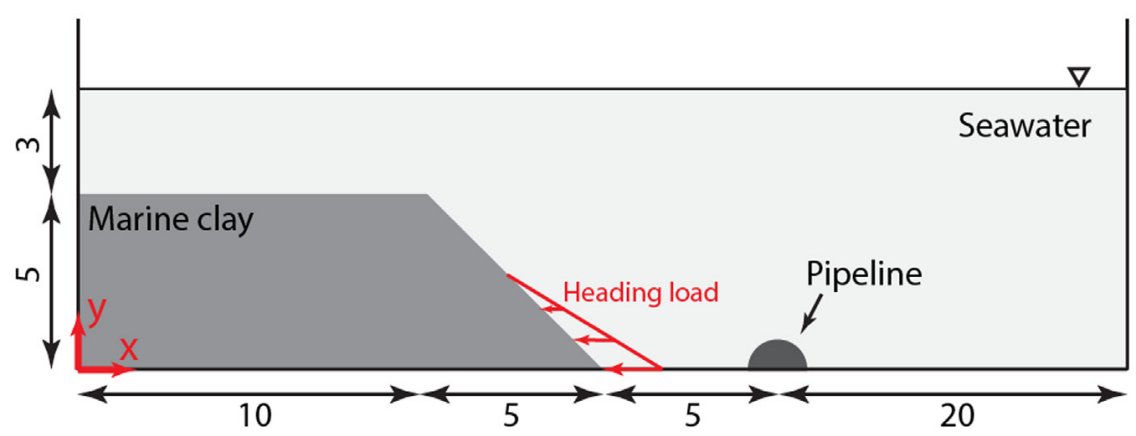

Fig. 13. Schematic illustration of an underwater slope near a subsea pipeline (Unit of length: m).
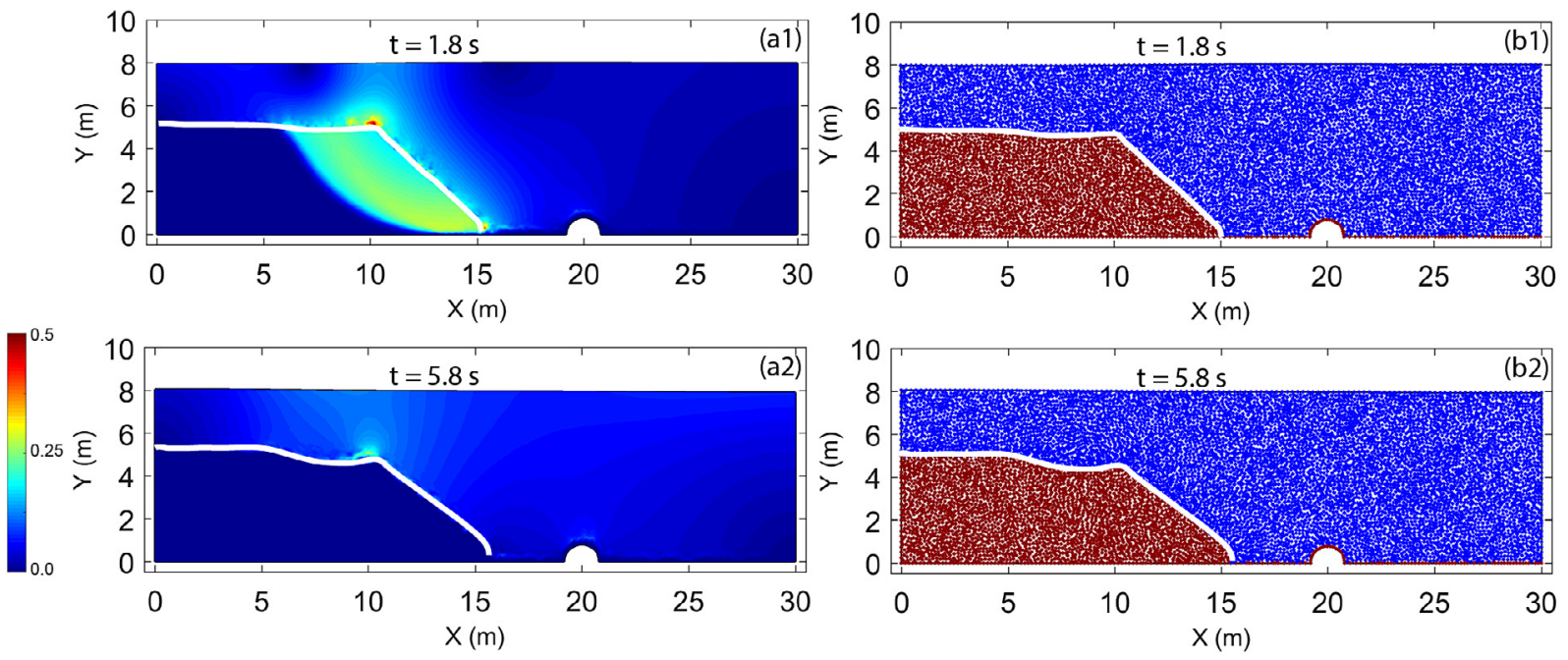

Fig. 14. Snapshots of the collapse process of the submarine landslide at different time instances from simulations without strain softening. Colours on the left figures are proportional to velocity $(\mathrm{m} / \mathrm{s})$ and figures on the right show the layers of the materials with blue and red colours representing seawater and marine clays, respectively (Unit of speed: $\mathrm{m} / \mathrm{s}$ ). (For interpretation of the references to colour in this figure legend, the reader is referred to the web version of this article.)

\subsection{Submarine landslides}

Last but not least, the possibility of the proposed unified formulation for modelling submarine landslides is presented via analysing underwater slope failure and its consequence. As shown in Fig. 13, a marine clay slope of height $5 \mathrm{~m}$ and length $5 \mathrm{~m}$ is $3 \mathrm{~m}$ under the water surface. A half-buried pipeline of diameter $1.6 \mathrm{~m}$ is located $5 \mathrm{~m}$ in front of the slope toe. It is supposed that permeability of marine clays is very low so that the slope can be simulated under undrained conditions. The marine clays are represented by the Tresca model with viscosity. The corresponding material parameters for the clay are as follows: Young's modulus $E=3 \times 10^{7} \mathrm{~Pa}$, Poisson's ratio $v=0.49$, density $\rho_{\mathrm{c}}$ $=1.75 \times 10^{3} \mathrm{~kg} / \mathrm{m}^{3}$, undrained shear strength $c_{\mathrm{u}}=6 \mathrm{kPa}$ and viscosity coefficient $\eta=50 \mathrm{~Pa}$ s. The density of seawater is $\rho_{\mathrm{w}}=1 \times 10^{3} \mathrm{~kg} / \mathrm{m}^{3}$ and the viscosity coefficient is $\eta=0.001 \mathrm{~Pa}$. The gravitational acceleration is $g=$ $-9.8 \mathrm{~m} / \mathrm{s}^{2}$. The surfaces of the seabed and the pipeline are assumed to be rough.

The slope was stable owing to the heading load which is then removed representing toe erosion. The factor of safety of the resulting slope is 0.90 implying unstability. The problem is simulated using the proposed approach. The characteristic mesh size is $0.02 \mathrm{~m}$ leading to a total of 19,452 elements (39,303 element nodes) for discretising the domains of marine clays and seawater. The time step used in the simulation is $\Delta t=5 \times 10^{-3} \mathrm{~s}$, and the simulation proceeds until the final deposit is obtained. As shown in Fig. 14, the failure of the slope is triggered due to the removing of the heading load. The mass in the front slides along a failure surface but at a relatively low speed in this case (Fig. 14(a1)). After a very limited deformation, the slope turns to be stable at a new position (Fig. 14(a2)). 


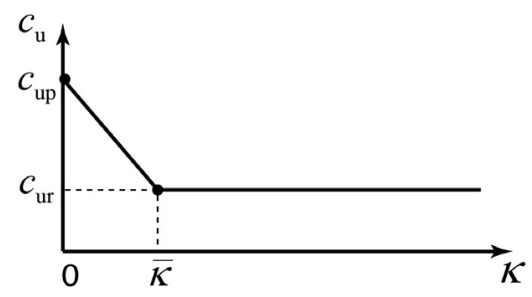

Fig. 15. Variation of the undrained shear strength $c_{\mathrm{u}}$ with equivalent deviatoric plastic strain represented by parameter $\kappa$.

Fig. 14(b1) and (b2) indicate the corresponding layers of seawater and marine clays for comparison. Throughout the process, no obvious tsunami is generated.

Notably, marine clay is normally sensitive which means its undrained strength decreases from a peak value $c_{\text {up }}$ to a residual one $c_{\mathrm{ur}}$ when the clay undergoes plastic deformation (see Fig. 15). It is reported in [8] that the sensitivity of marine clays, defined as $S_{\mathrm{t}}={ }_{{ }_{c_{\text {ur }}}}^{\text {up }}$, is normally moderate. Herein the problem is re-analysed with the strain-softening feature being taken into account. The peak undrained strength is $c_{\text {up }}=6 \mathrm{kPa}$ and the residual one is $c_{\text {ur }}=1.5 \mathrm{kPa}$, implying a moderate sensitivity $\left(S_{\mathrm{t}}=4\right)$. The reference equivalent deviatoric plastic strain $\bar{\kappa}$, which controls the rate of the decrease of the undrained strain is set to be 0.6. The complete process of the submarine landslides from the simulation is illustrated in Fig. 16. The distribution of the sliding speed is shown in Fig. 16(a) in which the white curves are the interface between the seawater and the clay drawn according to Fig. 16(b) where particles (mesh nodes) representing different materials are plotted. The same to the previous case, the removing of the heading load triggers the failure of the slope as shown in Fig. 16(a1) in which a shear band is expected along the failure surface. The clay evoked slides along the failure surface and towards the pipeline (Fig. 16(a2) and (b2)). At $t=6.0 \mathrm{~s}$, the pipeline is impacted by the sliding mass (Fig. 16(a3) and (b3)). When the evoked mass is far enough from the newly generated back scarp of the slope, a second failure occurs as shown in Fig. 16(a4) and (b4). This feature is very typical for slope failure in sensitive clays and is usually termed retrogressively progressive failure [66]. Eventually, the landslide reaches its final deposition as shown in Fig. 16(a5) and (b5). The failure of the underwater slope in this case generates a clear tsunami in the process (Fig. 16).

The effect of sensitivity of marine clays on the failure of a submarine slope is also investigated by using different St. Fig. 17 shows the final deposition of the landslides from the simulation with St equal to 1, 2, 3, and 4, respectively. As shown, the slope is more prone to fail when the sensitivity is large. Additionally, the sliding mass involved in each retrogressive collapse is much easier to be further decomposed when sensitivity is higher.

\section{Conclusions}

This paper recasts the finite element formulation for fluid dynamics and solid mechanics into a unified elastoviscoplastic formulation. This is achieved by employing the generalised Hellinger-Reissner variational principle. The governing equations for both the fluid dynamics and the solid mechanics are reformulated into a standard optimisation problem, namely a min-max program, which then can be transformed into a second-order cone programming problem and solved via advanced modern optimisation algorithm. In such a way, the coupling between the solid and the fluid can be completed in a monolithic fashion which is particularly important for modelling submarine landslides. The resulting formulation is implemented in the framework of the particle finite element method so that extreme deformation problems can be simulated without any mesh distortion issue. A number of benchmarks of both singlephase problems, involving Newtonian/Non-Newtonian flows or solids, and multi-phase problems, such as the model test on submarine landslide generated tsunamis, are simulated using the proposed approach. Comparisons between the simulation results with available data and analytical solutions are conducted where great agreements have been attained which verifies the proposed method. Last but not least, a model test is considered to illustrate the possibility of the proposed approach for modelling the consequences of submarine landslides including their direct threat to offshore infrastructure such as pipelines and their indirect threat via generating tsunamis. Sensitivity of the marine clays is also considered in this example with its effect on the failure of underwater slope being shown. 

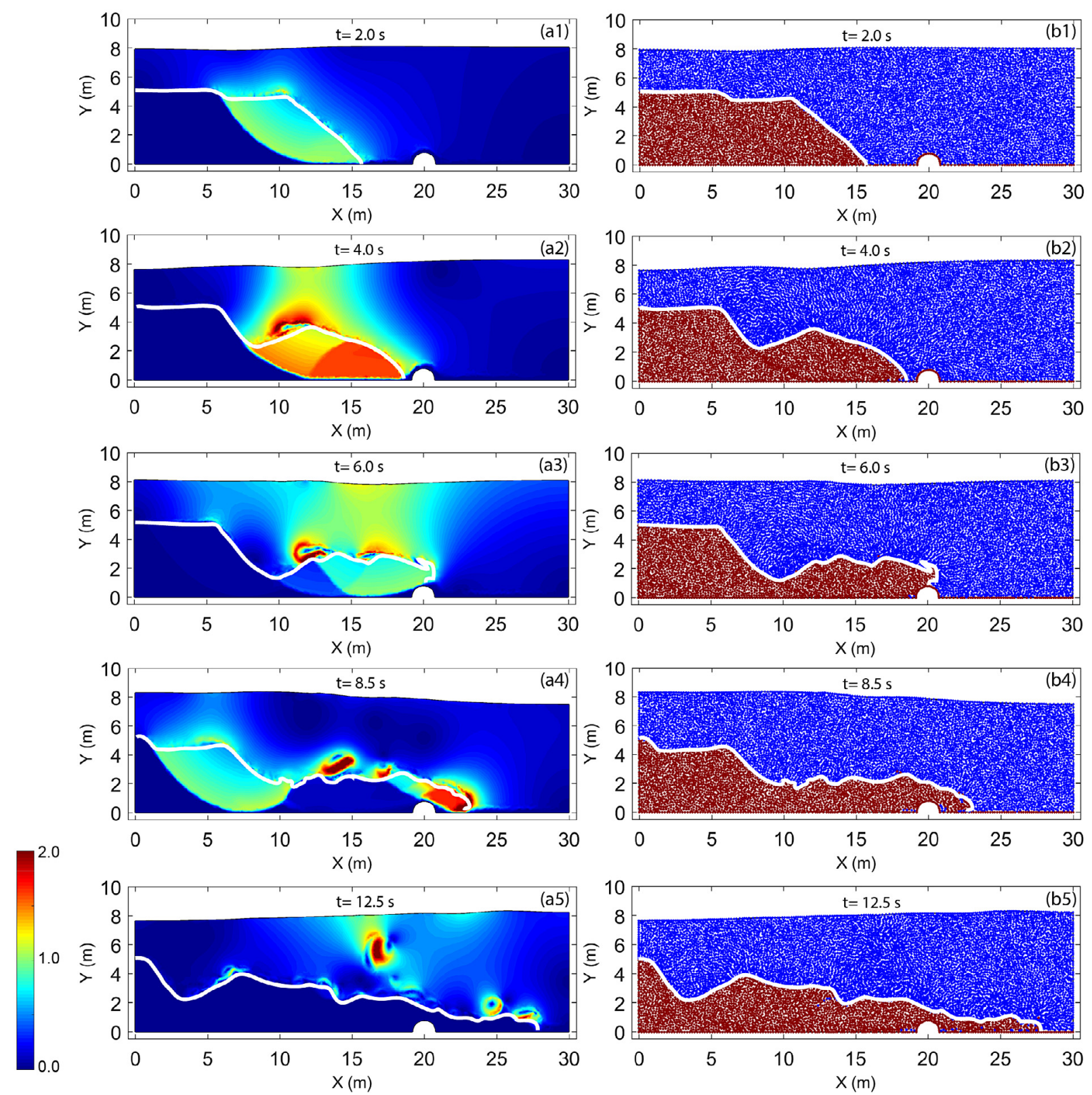

Fig. 16. Snapshots of the collapse process of the submarine landslide at different time instances from simulations with strain softening $(\mathrm{St}=4)$. Colours on the left figures are proportional to velocity $(\mathrm{m} / \mathrm{s})$ and figures on the right show the layers of the materials with blue and red colours representing seawater and marine clays, respectively (Unit of speed: $\mathrm{m} / \mathrm{s}$ ). (For interpretation of the references to colour in this figure legend, the reader is referred to the web version of this article.)

\section{Acknowledgement}

The authors wish to acknowledge the support of European Commission H2020 Marie Sklodowska-Curie Actions (MSCA) Individual Fellowship, Spain (Reference 744281; Proposal title "Towards Submarine Landslides and Their Consequences"). 

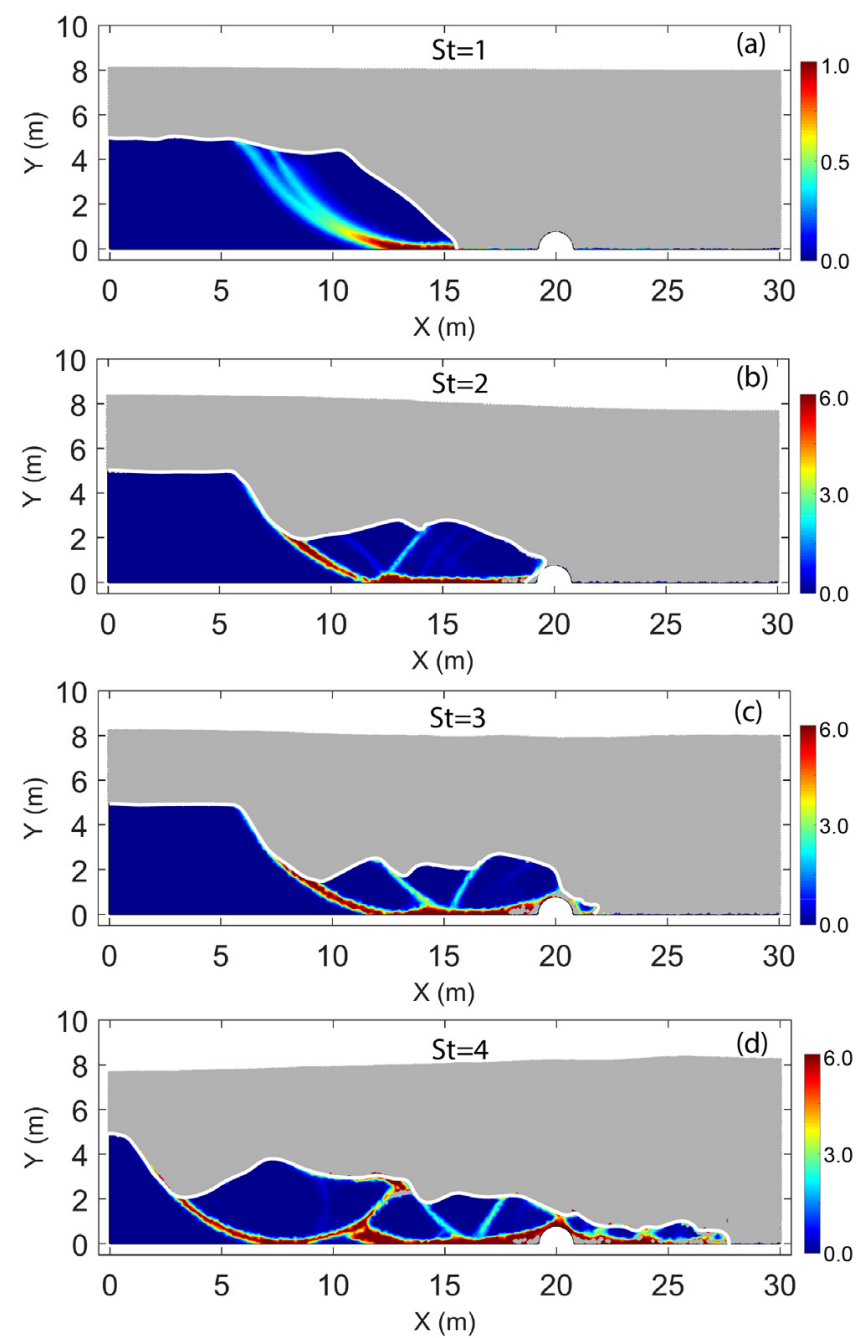

Fig. 17. Snapshots of final depositions of the submarine landslide from simulations with sensitivity of marine clays (a) $\mathrm{St}=1$, (b) $\mathrm{St}=2$, (c) $\mathrm{St}=$ 3 , and (d) $\mathrm{St}=4$. Colours are proportional to equivalent deviatoric plastic strain.

\section{Appendix}

In this study, the discretised principle (60) is transferred into the standard SOCP problem, namely the optimisation problem (1). The principle (60) is in a general form of

$$
\begin{array}{ll}
\min _{\mathbf{x}} & \mathbf{x}^{\mathrm{T}} \mathbf{Q} \mathbf{x}+\mathbf{c}^{\mathrm{T}} \mathbf{x} \\
\text { subject to } & \mathbf{A x}=\mathbf{b} \\
& F(\mathbf{x}) \leq 0
\end{array}
$$

and the relevant transformation is straightforward. Introducing an auxiliary variable $\mathrm{w}=\mathbf{x}^{\mathrm{T}} \mathbf{Q} \mathbf{x}$ and intermediate variables $\xi=\mathbf{Q}^{\underline{1}}{ }^{2} \mathbf{x}$, problem (A.1) can be re-written as

$$
\begin{array}{ll}
\min _{(\mathbf{x}, w, y, \boldsymbol{\xi})} & w+\mathbf{c}^{\mathrm{T}} \mathbf{x} \\
\text { subject to } & 2 w y \geq \boldsymbol{\xi}^{\mathrm{T}} \boldsymbol{\xi} \\
& \boldsymbol{\xi}=\mathbf{Q}^{\frac{1}{2}} \mathbf{x} ; \quad y=1 \\
& \mathbf{A x}=\mathbf{b} \\
& F(\mathbf{x}) \leq 0
\end{array}
$$


It is clear that optimisation problem (A.2) is of a linear objective function subject to linear equations, an inequality constraint (the first inequality) of a type of a rotated quadratic cone (3), and an inequality for yielding $F(\mathbf{x}) \leq 0$. Following this procedure, the principle (60) can be transferred as a standard SOCP problem

$$
\begin{aligned}
& \min _{\hat{x}^{\mathrm{c}} \mathrm{x}^{\mathrm{d}} \mathrm{m} x^{\mathrm{h}}} x_{\mathrm{n}+1}^{\mathrm{c}}+x_{\mathrm{n}+1}^{\mathrm{d}}+x_{\mathrm{n}+1}^{\mathrm{m}}+x_{\mathrm{n}+1}^{\mathrm{h}}+\Delta \hat{\boldsymbol{\sigma}}^{\mathrm{eT}} \mathbf{f}^{\mathrm{c}} \\
& \left(\hat{\boldsymbol{\sigma}}, \hat{\mathbf{\tau}}, \hat{\boldsymbol{\sigma}}^{\mathrm{e}}, \hat{\mathbf{r}}, \hat{\mathbf{k}}, x^{\mathrm{c}}, x^{\mathrm{d}}, x^{\mathrm{m}}, x^{\mathrm{h}},\right. \\
& \left.y^{\mathrm{c}}, y^{\mathrm{d}}, y^{\mathrm{m}}, y^{\mathrm{h}}, \boldsymbol{\xi}^{\mathrm{c}}, \boldsymbol{\xi}^{\mathrm{d}}, \boldsymbol{\xi}^{\mathrm{m}}, \boldsymbol{\xi}^{\mathrm{h}}\right)_{\mathrm{n}+1} \\
& \text { subject to } \\
& \mathbf{B}^{\mathrm{T}} \hat{\boldsymbol{\sigma}}_{\mathrm{n}+1}+\frac{1-\theta_{1}}{\theta_{1}} \mathbf{B}^{\mathrm{T}} \hat{\boldsymbol{\sigma}}_{\mathrm{n}}+\mathbf{A}^{\mathrm{T}} \hat{\mathbf{r}}_{\mathrm{n}+1}-\mathbf{f}^{\mathrm{e}}=\mathbf{0} \\
& \Delta \hat{\boldsymbol{\sigma}}^{\mathrm{e}}=\Delta \hat{\boldsymbol{\sigma}}-\Delta \hat{\boldsymbol{\tau}} \\
& \mathbf{C}^{\frac{1}{2}} \hat{\boldsymbol{\sigma}}_{\mathrm{n}+1}-\boldsymbol{\xi}_{\mathrm{n}+1}^{\mathrm{c}}=\mathbf{C}^{\frac{1}{2}} \hat{\boldsymbol{\sigma}}_{\mathrm{n}}, \quad \mathbf{D}^{\frac{1}{2}} \hat{\mathbf{r}}_{\mathrm{n}+1}-\boldsymbol{\xi}_{\mathrm{n}+1}^{\mathrm{d}}=\mathbf{D}^{\frac{1}{2}} \hat{\mathbf{r}}_{\mathrm{n}} \\
& \mathbf{M}^{\frac{1}{2}} \hat{\boldsymbol{\sigma}}_{\mathrm{n}+1}^{\mathrm{e}}-\boldsymbol{\xi}_{\mathrm{n}+1}^{\mathrm{m}}=\mathbf{M}^{\frac{1}{2}} \hat{\boldsymbol{\sigma}}_{\mathrm{n}}^{\mathrm{e}}, \mathbf{H}^{\frac{1}{2}} \hat{\boldsymbol{\kappa}}_{\mathrm{n}+1}^{\mathrm{e}}-\xi_{\mathrm{n}+1}^{\mathrm{h}}=\mathbf{H}^{\frac{1}{2}} \hat{\boldsymbol{\kappa}}_{\mathrm{n}}^{\mathrm{e}} \\
& y_{\mathrm{n}+1}^{\mathrm{c}}=1 ; \quad\left\{\left(x_{\mathrm{n}+1}^{\mathrm{c}}, y_{\mathrm{n}+1}^{\mathrm{c}}, \boldsymbol{\xi}_{\mathrm{n}+1}^{\mathrm{c}}\right) \in \mathrm{R}^{\mathrm{m}+2} \mid 2 x_{\mathrm{n}+1}^{\mathrm{c}} y_{\mathrm{n}+1}^{\mathrm{c}} \geq \xi_{\mathrm{n}+1}^{\mathrm{cT}} \xi_{\mathrm{n}+1}^{\mathrm{c}}\right\} \\
& y_{\mathrm{n}+1}^{\mathrm{d}}=1 ; \quad\left\{\left(x_{\mathrm{n}+1}^{\mathrm{d}}, y_{\mathrm{n}+1}^{\mathrm{d}}, \xi_{\mathrm{n}+1}^{\mathrm{d}}\right) \in \mathrm{R}^{\mathrm{m}+2} \mid 2 x_{\mathrm{n}+1}^{\mathrm{d}} y_{\mathrm{n}+1}^{\mathrm{d}} \geq \xi_{\mathrm{n}+1}^{\mathrm{dT}} \xi_{\mathrm{n}+1}^{\mathrm{d}}\right\} \\
& y_{\mathrm{n}+1}^{\mathrm{m}}=1 ; \quad\left\{\left(x_{\mathrm{n}+1}^{\mathrm{m}}, y_{\mathrm{n}+1}^{\mathrm{m}}, \boldsymbol{\xi}_{\mathrm{n}+1}^{\mathrm{m}}\right) \in \mathrm{R}^{\mathrm{m}+2} \mid 2 x_{\mathrm{n}+1}^{\mathrm{m}} y_{\mathrm{n}+1}^{\mathrm{m}} \geq \boldsymbol{\xi}_{\mathrm{n}+1}^{\mathrm{mT}} \xi_{\mathrm{n}+1}^{\mathrm{m}}\right\} \\
& y_{\mathrm{n}+1}^{\mathrm{h}}=1 ;\left\{\left(x_{\mathrm{n}+1}^{\mathrm{h}}, y_{\mathrm{n}+1}^{\mathrm{h}}, \xi_{\mathrm{n}+1}^{\mathrm{h}}\right) \in \mathrm{R}^{\mathrm{m}+2} \mid 2 x_{\mathrm{n}+1}^{\mathrm{h}} y_{\mathrm{n}+1}^{\mathrm{h}} \geq \xi_{\mathrm{n}+1}^{\mathrm{hT}} \xi_{\mathrm{n}+1}^{\mathrm{h}}\right\} \\
& F_{j}\left(\hat{\boldsymbol{\tau}}_{\mathrm{n}+1}, \hat{\boldsymbol{\kappa}}_{\mathrm{n}+1}\right) \leq 0, j=1,2, \ldots, N_{G}
\end{aligned}
$$

Note that numerous yield criterion functions have been expressed as cone constraints successfully including the Mohr-Coulomb/Tresca model, the Drucker-Prager/von Mises model, the CamClay model etc. We refer the reader to $[32,67,68]$ for more details. As a standard SOCP problem, the optimisation problem (A.3) can be solved using the primal-dual interior point method which is a standard approach. We refer the reader to [60] for more details of this solution algorithm. The efficiency of the primal-dual interior point method for the SOCP problem has been discussed in $[26,42,60]$. Moreover, the convergence property of the primal-dual interior point method and its variant for the SOCP problem has also been analysed mathematically [21,69]. It has been proven via mathematical analysis in [21] that the primal-dual interior point method possesses strong global and local convergence property for the SOCP problem. In this study, the high-performance optimisation engine MOSEK [60] which supports the primal-dual interior point method with parallel computing is adopted for solutions.

\section{References}

[1] D.R. Tappin, P. Watts, G.M. McMurtry, Y. Lafoy, T. Matsumoto, The Sissano, Papua New Guinea tsunami of 1998 - offshore evidence on the source mechanism, Mar. Geol. 175 (1) (2001) 1-23.

[2] L. Carter, R. Gavey, P.J. Talling, J.T. Liu, Insights into submarine geohazards from breaks in subsea telecommunication cables, Oceanography 27 (2) (2014) 58-67.

[3] T. Capone, A. Panizzo, J.J. Monaghan, SPH modelling of water waves generated by submarine landslides, J. Hydraul. Res. 48 (Sup1) (2010) 80-84.

[4] S. Rzadkiewicz, C. Mariotti, P. Heinrich, Numerical simulation of submarine landslides and their hydraulic effects, J. Waterw. Port Coastal Ocean Eng. 123 (4) (1997) 149-157.

[5] P.H. Heinrich, A. Piatanesi, H. Hébert, Numerical modelling of tsunami generation and propagation from submarine slumps: the 1998 Papua New Guinea event, Geophys. J. Int. 145 (1) (2001) 97-111.

[6] I. Didenkulova, I. Nikolkina, E. Pelinovsky, N. Zahibo, Tsunami waves generated by submarine landslides of variable volume: analytical solutions for a basin of variable depth, Nat. Hazards Earth Syst. Sci. 10 (11) (2010) 2407-2419.

[7] D. Wang, M.F. Randolph, D.J. White, A dynamic large deformation finite element method based on mesh regeneration, Comput. Geotech. 54 (2013) 192-201.

[8] R. Dey, B. Hawlader, R. Phillips, K. Soga, Modeling of large-deformation behaviour of marine sensitive clays and its application to submarine slope stability analysis, Can. Geotech. J. 53 (7) (2016) 1138-1155.

[9] R. Dey, B.C. Hawlader, R. Phillips, K. Soga, Numerical modelling of submarine landslides with sensitive clay layers, Géotechnique 66 (6) (2016) 454-468.

[10] B.G. McAdoo, L.F. Pratson, D.L. Orange, Submarine landslide geomorphology, US continental slope, Mar. Geol. 169 (1) (2000) 103-136.[11] F.V.D. Blasio, L. Engvik, C.B. Harbitz, A. Elverhøi, Hydroplaning and submarine debris flows, J. Geophys. Res. Oceans 109 (C1) (2004).[12] P. Gauer, T.J. Kvalstad, C.F.

Forsberg, P. Bryn, K. Berg, The last phase of the Storegga Slide: simulation of retrogressive slide dynamics and

comparison with slide-scar morphology, Mar. Pet. Geol. 22 (1) (2005) 171-178.

[13] A. Franci, E. Oñate, J.M. Carbonell, Unified Lagrangian formulation for solid and fluid mechanics and FSI problems, Comput. Methods

Appl.Mech. Engrg. 298 (2016) 520-547. 
[14] U. Langer, H. Yang, Robust and efficient monolithic fluid-structure-interaction solvers, Internat. J. Numer. Methods Engrg. 108 (4) (2016) 303-325.

[15] J. Degroote, Partitioned simulation of fluid-structure interaction, Arch. Comput. Methods Eng. 20 (3) (2013) 185-238.

[16] R. Rossi, E. Oñate, Analysis of some partitioned algorithms for fluid-structure interaction, Eng. Comput. 27 (1) (2010) 20-56.

[17] P.B. Ryzhakov, R. Rossi, S.R. Idelsohn, E. Oñate, A monolithic Lagrangian approach for fluid-structure interaction problems, Comput. Mech. 46 (6) (2010) 883-899.

[18] L. Ulrich, Y. Huidong, Robust and efficient monolithic fluid-structure-interaction solvers, Internat. J. Numer. Methods Engrg. 108 (4) (2016) $303-325$.

[19] B. Hübner, E. Walhorn, D. Dinkler, A monolithic approach to fluid-structure interaction using space-time finite elements, Comput. Methods Appl. Mech. Engrg. 193 (23) (2004) 2087-2104.

[20] C. Michler, S.J. Hulshoff, E.H. van Brummelen, R. de Borst, A monolithic approach to fluid-structure interaction, Comput. \& Fluids 33 (5) (2004) 839-848

[21] A.L. Tits, A. Wächter, S. Bakhtiari, T.J. Urban, C.T. Lawrence, A primal-dual interior-point method for nonlinear programming with strong global and local convergence properties, SIAM J. Optim. 14 (1) (2003) 173-199.

[22] F. Alizadeh, J.-P.A. Haeberly, M.L. Overton, Primal-dual interior-point methods for semidefinite programming: Convergence rates, stability and numerical results, SIAM J. Optim. 8 (3) (1998) 746-768.

[23] A.J. Abbo, A.V. Lyamin, S.W. Sloan, J.P. Hambleton, A C2 continuous approximation to the Mohr-Coulomb yield surface, Int. J. Solids Struct. 48 (21) (2011) 3001-3010.

[24] A. Franci, X. Zhang, 3D numerical simulation of free-surface Bingham fluids interacting with structures using the PFEM, J. Non-Newton. Fluid Mech. 259 (2018) 1-15.

[25] K. Krabbenhøft, A. Lyamin, S. Sloan, Formulation and solution of some plasticity problems as conic programs, Int. J. Solids Struct. 44 (5) (2007) 1533-1549.

[26] J. Bleyer, M. Maillard, P. de Buhan, P. Coussot, Efficient numerical computations of yield stress fluid flows using second-order cone programming, Comput. Methods Appl. Mech. Engrg. 283 (0) (2015) 599-614.

[27] C.V. Le, H. Nguyen-Xuan, H. Nguyen-Dang, Upper and lower bound limit analysis of plates using FEM and second-order cone programming, Comput. Struct. 88 (1-2) (2010) 65-73.

[28] A. Makrodimopoulos, C.M. Martin, Upper bound limit analysis using simplex strain elements and second-order cone programming, Int. J. Numer. Anal. Methods Geomech. 31 (6) (2007) 835-865.

[29] S. Yu, X. Zhang, S.W. Sloan, A 3D upper bound limit analysis using radial point interpolation meshless method and second-order cone programming, Internat. J. Numer. Methods Engrg. 108 (13) (2016) 1686-1704.

[30] K. Yonekura, Y. Kanno, Second-order cone programming with warm start for elastoplastic analysis with von Mises yield criterion, Optim. Eng. 13 (2) (2012) 181-218.

[31] X. Zhang, D. Sheng, S.W. Sloan, J. Bleyer, Lagrangian modelling of large deformation induced by progressive failure of sensitive clays with elastoviscoplasticity, Internat. J. Numer. Methods Engrg. 112 (8) (2017) 963-989.

[32] X. Zhang, D. Sheng, S.W. Sloan, K. Krabbenhoft, Second-order cone programming formulation for consolidation analysis of saturated porous media, Comput. Mech. 58 (1) (2016) 29-43.

[33] K.-W. Lim, K. Krabbenhoft, J.E. Andrade, A contact dynamics approach to the Granular Element Method, Comput. Methods Appl. Mech. Engrg. 268 (2014) 557-573

[34] K. Krabbenhoft, A.V. Lyamin, C. Vignes, Computational plasticity algorithm for particle dynamics simulations, Comput. Part. Mech. 5 (1) (2018) 103-111.

[35] K. Krabbenhoft, J. Huang, M.V. da Silva, A.V. Lyamin, Granular contact dynamics with particle elasticity, Granular Matter 14 (5) (2012) $607-619$.

[36] J. Meng, J. Huang, C. Yao, D. Sheng, A discrete numerical method for brittle rocks using mathematical programming, Acta Geotech. (2017).[37] J. Meng, J.

Huang, S.W. Sloan, D. Sheng, Discrete modelling jointed rock slopes using mathematical programming methods, Comput. Geotech. 96 (2018) 189-202.

[38] A. Marly, P. Vigneaux, Augmented Lagrangian simulations study of yield-stress fluid flows in expansion-contraction and comparisons with physical experiments, J. Non-Newton. Fluid Mech. 239 (2017) 35-52.

[39] L. Muravleva, E. Muravleva, G.C. Georgiou, E. Mitsoulis, Numerical simulations of cessation flows of a Bingham plastic with the augmented Lagrangian method, J. Non-Newton. Fluid Mech. 165 (9) (2010) 544-550.

[40] Y. Dimakopoulos, G. Makrigiorgos, G.C. Georgiou, J. Tsamopoulos, The PAL (Penalized Augmented Lagrangian) method for computing viscoplastic flows: A new fast converging scheme, J. Non-Newton. Fluid Mech. 256 (2018) 23-41.

[41] R.R. Huilgol, Variational inequalities in the flows of yield stress fluids including inertia: Theory and applications, Phys. Fluids 14 (3) (2002) 1269-1283.

[42] J. Bleyer, Advances in the simulation of viscoplastic fluid flows using interior-point methods, Comput. Methods Appl. Mech. Engrg. 330 (2018) 368-394.

[43] K. Krabbenhoft, A.V. Lyamin, S.W. Sloan, P. Wriggers, An interior-point algorithm for elastoplasticity, Internat. J. Numer. Methods Engrg. 69 (3) (2007) 592-626.

[44] E. Oñate, S.R. Idelsohn, F. Del Pin, R. Aubry, The particle finite element method - an overview, Int. J. Comput. Math. 01 (02) (2004) 267-307.[45] H

Edelsbrunner, E.P. Mücke, Three-dimensional alpha shapes, ACM Trans. Graph. 13 (1) (1994) 43-72.

[46] Y. Hu, M.F. Randolph, A practical numerical approach for large deformation problems in soil, Int. J. Numer. Anal. Methods Geomech. 22 (5) (1998) 327-350 
[47] X. Zhang, K. Krabbenhoft, D. Sheng, Particle finite element analysis of the granular column collapse problem, Granular Matter 16 (4) (2014) 609-619.

[48] S. Idelsohn, M. Mier-Torrecilla, E. Oñate, Multi-fluid flows with the Particle Finite Element Method, Comput. Methods Appl. Mech. Engrg. 198 (33-36) (2009) 2750-2767.

[49] M. Cremonesi, A. Frangi, U. Perego, A Lagrangian finite element approach for the analysis of fluid-structure interaction problems, Internat. J. Numer. Methods Engrg. 84 (5) (2010) 610-630.

[50] M. Zhu, M.H. Scott, Improved fractional step method for simulating fluid-structure interaction using the PFEM, Internat. J. Numer. Methods Engrg. 99 (12) (2014) 925-944.

[51] X. Zhang, Y. Ding, D. Sheng, S.W. Sloan, W. Huang, Quasi-static collapse of two-dimensional granular columns: insight from continuum modelling, Granular Matter 18 (3) (2016) 1-14.

[52] C. Dávalos, J. Cante, J.A. Hernández, J. Oliver, On the numerical modeling of granular material flows via the Particle Finite Element Method (PFEM), Int. J. Solids Struct. 71 (2015) 99-125.

[53] W. Zhang, W. Yuan, B. Dai, Smoothed particle finite-element method for large-deformation problems in geomechanics, Int. J. Geomech. 18 (4) (2018) 04018010

[54] M. Cremonesi, L. Ferrara, A. Frangi, U. Perego, Simulation of the flow of fresh cement suspensions by a Lagrangian finite element approach, J. Non-Newton. Fluid Mech. 165 (23-24) (2010) 1555-1563.

[55] L. Monforte, M. Arroyo, J.M. Carbonell, A. Gens, Numerical simulation of undrained insertion problems in geotechnical engineering with the Particle Finite Element Method (PFEM), Comput. Geotech. 82 (2017) 144-156.

[56] X. Zhang, K. Krabbenhoft, D. Pedroso, A. Lyamin, D. Sheng, M.V. Da Silva, D. Wang, Particle finite element analysis of large deformation and granular flow problems, Comput. Geotech. 54 (2013) 133-142.

[57] X. Zhang, K. Krabbenhoft, D. Sheng, W. Li, Numerical simulation of a flow-like landslide using the particle finite element method, Comput. Mech. 55 (1) (2015) 167-177.

[58] M. Cremonesi, F. Ferri, U. Perego, A basal slip model for Lagrangian finite element simulations of 3D landslides, Int. J. Numer. Anal. Methods Geomech. 41 (1) (2017) 30-53.

[59] F. Salazar, J. Irazábal, A. Larese, E. Oñate, Numerical modelling of landslide-generated waves with the particle finite element method (PFEM) and a non-Newtonian flow model, Int. J. Numer. Anal. Methods Geomech. 40 (6) (2016) 809-826.

[60] E.D. Andersen, C. Roos, T. Terlaky, On implementing a primal-dual interior-point method for conic quadratic optimization, Math. Program. 95 (2) (2003) 249-277.

[61] S. Shao, E.Y.M. Lo, Incompressible SPH method for simulating Newtonian and non-Newtonian flows with a free surface, Adv. Water Resour 26 (7) (2003) 787-800

[62] N. Nomeritae, E. Daly, S. Grimaldi, H.H. Bui, Explicit incompressible SPH algorithm for free-surface flow modelling: A comparison with weakly compressible schemes, Adv. Water Resour. 97 (2016) 156-167.

[63] R.B. Bird, R.C. Armstrong, O. Hassager, Dynamics of Polymetric Liquids Vol. Fluid Mechsnics, Wiley-Intersceience, New York, 1987.

[64] H.H. Bui, R. Fukagawa, K. Sako, S. Ohno, Lagrangian meshfree particles method (SPH) for large deformation and failure flows of geomaterial using elastic-plastic soil constitutive model, Int. J. Numer. Anal. Methods Geomech. 32 (12) (2008) 1537-1570.

[65] S.A. Rzadkiewicz, C. Mariotti, P. Heinrich, Numerical simulation of submarine landslides and their hydraulic effects, J. Waterw. Port Coastal Ocean Eng. 123 (4) (1997) 149-157.

[66] A. Locat, S. Leroueil, S. Bernander, D. Demers, H.P. Jostad, L. Ouehb, Progressive failures in eastern Canadian and Scandinavian sensitive clays, Can. Geotech. J. 48 (11) (2011) 1696-1712.

[67] K. Krabbenhoft, A.V. Lyamin, Computational Cam clay plasticity using second-order cone programming, Comput. Methods Appl. Mech. Eng. 209-212 (0) (2012) 239-249.

[68] A. Makrodimopoulos, Remarks on some properties of conic yield restrictions in limit analysis, Int. J. Numer. Methods Biomed. Eng. 26 (11) (2010) 1449-1461.

[69] H. Yamashita, H. Yabe, A primal-dual interior point method for nonlinear optimization over second-order cones, Optim. Methods Softw. 24 (3) (2009) 407-426. 